\title{
JMJD1C is required for the survival of acute myeloid leukemia by functioning as a coactivator for key transcription factors
}

\author{
Mo Chen, ${ }^{1}$ Nan Zhu, ${ }^{2,6}$ Xiaochuan Liu, ${ }^{3}$ Benoit Laurent, ${ }^{4,5}$ Zhanyun Tang, ${ }^{1}$ Rowena Eng, ${ }^{2}$ Yang Shi, ${ }^{4,5}$ \\ Scott A. Armstrong, ${ }^{2}$ and Robert G. Roeder ${ }^{1}$ \\ ${ }^{1}$ Laboratory of Biochemistry and Molecular Biology, The Rockefeller University, New York, New York 10065, USA; ${ }^{2}$ Human \\ Oncology and Pathogenesis Program, Memorial Sloan-Kettering Cancer Center, New York, New York 10065, USA; ${ }^{3}$ Department of \\ Microbiology, Biochemistry, and Molecular Genetics, Rutgers University, Newark, New Jersey 07103, USA; ${ }^{4}$ Division of Newborn \\ Medicine, Epigenetics Program, Department of Medicine, Boston Children's Hospital, Boston, Massachusetts 02115, USA; \\ ${ }^{5}$ Department of Cell Biology, Harvard Medical School, Boston, Massachusetts 02115, USA
}

RUNX1-RUNX1T1 (formerly AML1-ETO), a transcription factor generated by the t(8;21) translocation in acute myeloid leukemia (AML), dictates a leukemic program by increasing self-renewal and inhibiting differentiation. Here we demonstrate that the histone demethylase JMJD1C functions as a coactivator for RUNX1-RUNX1T1 and is required for its transcriptional program. JMJD1C is directly recruited by RUNX1-RUNX1T1 to its target genes and regulates their expression by maintaining low H3K9 dimethyl (H3K9me2) levels. Analyses in JMJD1C knockout mice also establish a JMJD1C requirement for RUNX1-RUNX1T1's ability to increase proliferation. We also show a critical role for JMJD1C in the survival of multiple human AML cell lines, suggesting that it is required for leukemic programs in different AML cell types through its association with key transcription factors.

[Keywords: AML1-ETO; acute myeloid leukemia; HEB; histone demethylase; LYL1; transcription regulation]

Supplemental material is available for this article.

Received June 11, 2015; revised version accepted September 29, 2015.

Hematopoiesis is controlled by the differential expression of key transcription factors that act cooperatively to maintain a well-orchestrated balance of hematopoietic stem cell (HSC) self-renewal and differentiation (Wilson et al. 2010). These functions of transcription factors are frequently dysregulated in leukemia by chromosomal translocations, mutations, or aberrant expression and lead to abnormal self-renewal (Look 1997). RUNX1-RUNX1T1 (commonly referred to as acute myeloid leukemia 1 [AML1]-ETO and referred to here as AE), generated by the $\mathrm{t}(8 ; 21)$ translocation, occurs in $\sim 15 \%$ of AML cases (Look 1997; Kaspers and Zwaan 2007) and acts to enhance self-renewal and inhibit myeloid differentiation.

AE consists of the DNA-binding domain (RUNT) of the hematopoietic transcription factor RUNX1 (also known as AML1) (Meyers et al. 1993) and almost the entire ETO corepressor. Several lines of evidence from in vivo studies suggest that AE plays a role in leukemia stem cells. AE RNA transcripts are found in hematopoietic cells of nonmyeloid lineages in some patients, suggesting

\footnotetext{
${ }^{6}$ Present address: Blood Research Institute, Blood Center of Wisconsin, Milwaukee, Wisconsin 53226, USA.

Corresponding author: roeder@rockefeller.edu

Article is online at http://www.genesdev.org/cgi/doi/10.1101/gad.267278. 115 .
}

that the AE fusion protein is present in the multipotent progenitor cells (Miyamoto et al. 1997). Consistent with various earlier studies (Miyamoto et al. 1997; Mulloy et al. 2002, 2003), recent studies of an inducible AE mouse model showed that leukemic granulocyte macrophage progenitors (GMPs) resulting from AE expression have acquired self-renewal abilities (Cabezas-Wallscheid et al. 2013). In addition, it has been shown that AE can promote self-renewal of mouse and human hematopoietic stem and progenitor cells (HSPCs) (Higuchi et al. 2002; Mulloy et al. 2003). However, since the complete AE alone is incapable of generating leukemia in mice, most studies in mice have used either the C-terminal-truncated AE designated AE9a (Yan et al. 2006), a full-length AE bearing several point mutants (DeKelver et al. 2013), or wild-type AE in combination with a gain-of-function mutation in a tyrosine kinase such as FLT3 (Schessl et al. 2005) or c-KIT (Wang et al. 2011b).

(C) 2015 Chen et al. This article is distributed exclusively by Cold Spring Harbor Laboratory Press for the first six months after the full-issue publication date (see http://genesdev.cshlp.org/site/misc/terms.xhtml). After six months, it is available under a Creative Commons License (Attribution-NonCommercial 4.0 International), as described at http:// creativecommons.org/licenses/by-nc/4.0/. 
Like many transcription factors, and depending on the target gene, AE can act as either an activator or a repressor. Mechanistically, earlier studies mainly focused on the repressor function of AE because ETO had been shown to interact with several (co)repressors such as NCOR and HDACs (Nimer and Moore 2004; Yan et al. 2004) and stably interact with E proteins to prevent p300 binding (Zhang et al. 2004). However, possible mechanisms of transcriptional activation by AE were not revealed until recently. Thus, two studies reported that AE interacts with coactivators p300 (Wang et al. 2011a) and PRMT1 (Shia et al. 2012), whereas a study from our laboratory showed that AE forms a stable complex (AETFC) with multiple hematopoietic transcription factors/cofactors that include CBF $\beta$ (Gorczynski et al. 2007; Park et al. 2009), E proteins (E2A and HEB), oncoprotein LYL1, and adaptor proteins LMO2 and LDB1 (Sun et al. 2013). Since the targeting of interactions between transcription factors and cofactors has been proposed to have therapeutic potential (Cerchietti et al. 2009, 2010), we sought to identify additional AE cofactors that may thus serve as more tractable therapeutic targets.

Histone-modifying enzymes and modified histonebinding proteins, which serve as potential therapeutic targets because of their enzymatic sites and binding pockets, have been implicated in leukemia (Deshpande et al. 2012; Popovic and Licht 2012). The H3K79 methyltransferase DOT1L accounts for aberrant H3K79 methylation levels in MLL translocation-related leukemias (Bernt et al. 2011), and inhibition of the histone demethylase LSD1 in combination with all-trans retinoic acid (ATRA) can induce differentiation of leukemic cells (Schenk et al. 2012). The bromodomain protein BRD4, which recognizes acetylated lysines on histones, has also been identified as a therapeutic target (Filippakopoulos et al. 2010; Zuber et al. 2011). Importantly, several small molecule inhibitors have been developed against these proteins. These include JQ1 (Filippakopoulos et al. 2010; Zuber et al. 2011) and i-BET151 (Dawson et al. 2011), inhibitors of BRD4, and the DOT1L inhibitor EPZ004777 (Daigle et al. 2011).

As a key transcription factor in $\mathrm{t}(8,21)$ leukemias, $\mathrm{AE}$ is thought to control cancer cell state through interactions with genomic elements and subsequent recruitment of cofactors (e.g., chromatin remodeling and histone-modifying enzymes) that regulate gene expression. Several studies have defined the genomic localization of $\mathrm{AE}$ and several corresponding histone modifications in AEexpressing cells (Martens et al. 2012; Ptasinska et al. 2012; Saeed et al. 2012). These studies reported a decrease of $\mathrm{H} 3 / \mathrm{H} 4$ acetylation levels for a subset of AE-bound genes, suggesting a correlation between AE occupancy and the resulting changes of histone modifications by recruitment of HDACs. However, these studies failed to directly connect AE or other potentially associated transcription factors to histone modification changes and did not analyze the possible mechanism of gene activation by AE.

In an effort to understand the molecular mechanisms underlying transcriptional activation by $\mathrm{AE}$ and search for potential therapeutic candidates, we performed an un- biased proteomic analysis of AE-associated proteins in leukemic (patient-derived) Kasumi-1 cells. In this study, we found that the histone lysine demethylase JMJD1C interacts directly with AE both in cells and in vitro. JMJD1C was originally identified as a ligand-dependent interacting partner of thyroid hormone (Lee et al. 1995) and androgen (Wolf et al. 2007) receptors and contains conserved JmjC and zinc finger domains that are jointly required for its demethylase activity (Yamane et al. 2006). Reported substrates include H3K9 dimethyl (H3K9me2) (Kim et al. 2010) and MDC1, a protein involved in DNA damage repair (Watanabe et al. 2013). In our study, we demonstrate that JMJD1C is recruited by AE to target genes, that depletion of AE or JMJD1C leads to an increase of H3K9me2 levels on these target genes, and that JMJD1C is required for survival of multiple AML cells, possibly through its interaction with key transcription factors in these human AML cell lines.

\section{Results}

\section{IMJD1C and AETFC interact in vivo and in vitro}

Our recent study (Sun et al. 2013) demonstrated that AE forms a stable complex (AETFC) with several hematopoietic transcription factors. In order to further understand the molecular mechanism by which these transcription factors activate AE target genes in the context of $t(8 ; 21)$ leukemia, we used an unbiased immunoprecipitation proteomic analysis to identify candidate AE coactivators. Given that coactivators usually interact with transcription factors in a dynamic manner, the purification was performed under less stringent conditions than those used in our earlier study. In order to reduce nonspecific binding and preserve cell viability, we established a Kasumi-1 cell line (Kasumi-1-HF-AE) that can be induced to express HA-Flag-AE at a level similar to that of the endogenous AE (Supplemental Fig. S1A). Nuclear extracts (NEs) derived from control and Kasumi-1-HF-AE cells were used in a Flag-HA tandem purification protocol. Bound proteins were resolved by SDS-PAGE and analyzed by mass spectrometry (Fig. 1A).

This analysis identified the intact AETFC (Fig. 1A, right; Supplemental Table S1) and known corepressors or components of corepressor complexes that include HDACs, RBBP4, MTA2, and Co-REST (Lee et al. 2005), suggesting possible roles of these complexes in AE-dependent gene repression. In search of potential transcriptional coactivators, we found novel AE-associated proteins, including a presumptive complex consisting of PARP1, DNAPKcs, Ku70, and Ku80 (Fig. 1B; data now shown). Another very interesting hit was the H3K9me1/2 demethylase JMJD1C. H3K9me1 and H3K9me2 are modifications associated with transcriptional repression (Black et al. 2012). Notably, H3K9me2 is absent in primitive hematopoietic cells but appears at CpG islands in human HSPCs and expands during differentiation /Chen et al. 2012). Taking into account the correlation of H3K9me2 levels in normal hematopoietic development, we hypothesized that JMJD1C may be recruited by $\mathrm{AE}$ 
A
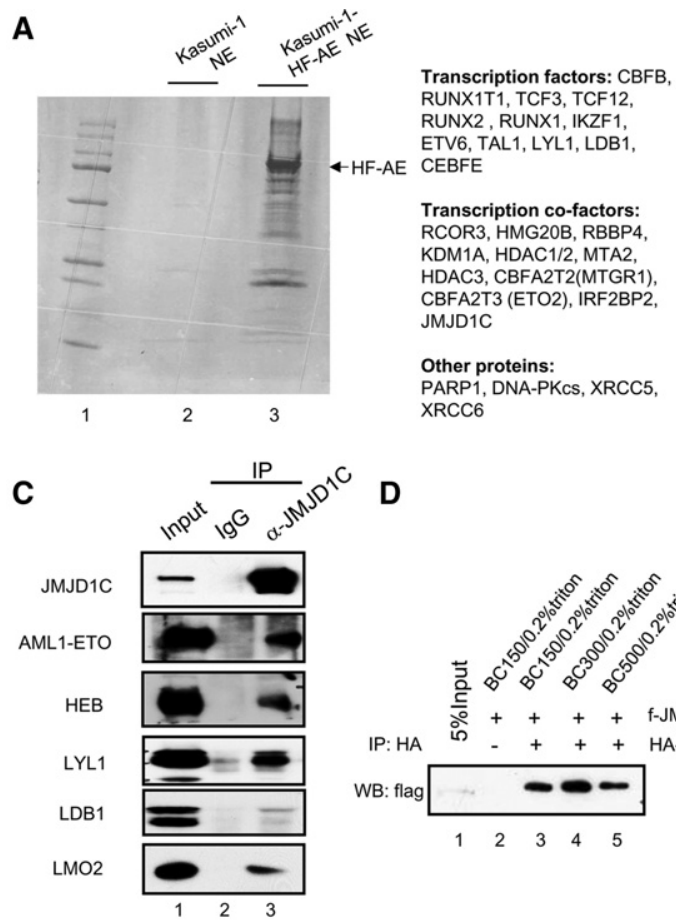

$\mathbf{F}$

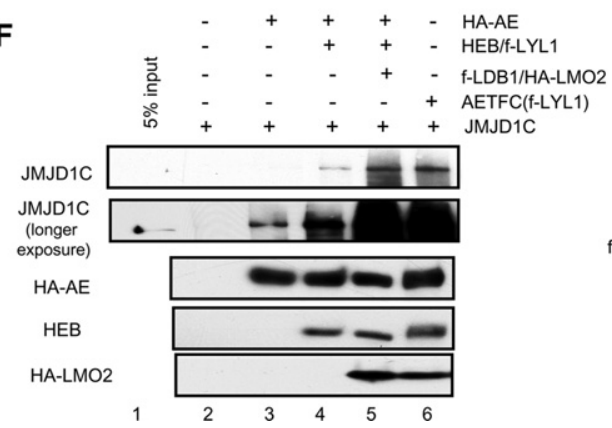

B

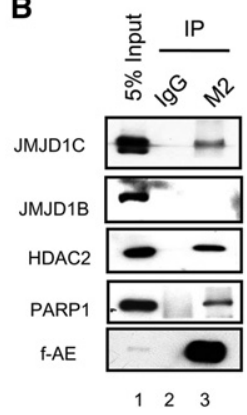

E

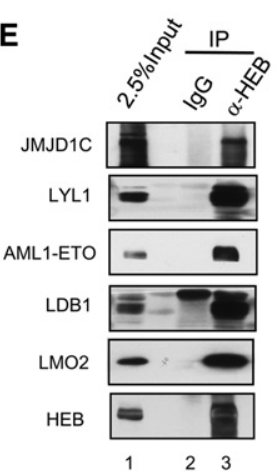

G

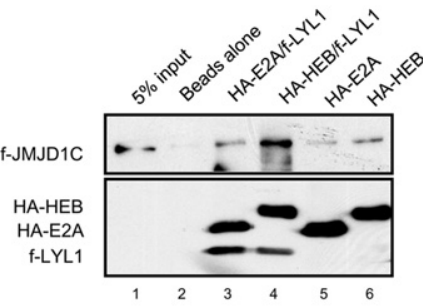

Figure 1. JMJD1C interacts with AETFC in vivo and in vitro. (A, left panel) SDS-PAGE and Commassie staining of HF-AE and associated proteins isolated from Kasumi-1 NE. Immunoprecipitation was performed using NE from Kasumi-1 cells either without (lane 2) or with (lane 3) HF-AE expression. (Lane 1) Protein markers. (Right panel), Identified proteins from this purification. $(B)$ Coimmunoprecipitation and immunoblot confirmation of AE-associated proteins. HF-AE was immunoprecipitated with M2 agarose, and associated proteins were blotted with antibodies to proteins indicated at the left. $(C)$ Immunoprecipitation of JMJD1C to confirm AETFC association. Bound proteins were detected with antibodies shown at the left. $(D)$ Direct interaction between High Five cell-purified f-JMJD1C and HA-AE proteins. Purified HA-AE was immobilized on anti-HA agarose and then incubated with purified f-JMJD1C. Samples were washed with buffers containing increasing amounts of $\mathrm{KCl}$. (E) Immunoprecipitation of HEB to confirm JMJD1C association. Bound proteins were detected with antibodies to proteins shown at the left. $(F)$ JMJD1C interacts more strongly with AETFC than with individual components of the complex. (Top) f-JMJD1C was incubated with the indicated bait proteins immobilized on anti-HA agarose beads. Individual proteins detected by immunoblot are indicated at the left of each blot. Anti$\mathrm{HA}$ antibody was used to detect $\mathrm{AE}$ and $\mathrm{LMO}$, anti-Flag antibody was used to detect JMJD1C, and anti-HEB antibody was used to detect HEB. (G) Direct interaction between f-JMJD1C and E proteins. (Top) Purified E proteins were incubated with f-JMJD1C, and anti-Flag immunoprecipitates were analyzed by immunoblot with antibodies to the proteins indicated at the left. and thereby play a role in maintaining low levels of $\mathrm{H} 3 \mathrm{~K} 9 \mathrm{me} 2$ at $\mathrm{AE}$ target genes.

We next confirmed that JMJD1C and AE physically associate in Kasumi-1 cells by reciprocal coimmunoprecipitation experiments (Fig. 1B,C). This analysis used a custom-made rabbit polyclonal JMJD1C antibody that specifically recognizes JMJD1C, but not JMJD1A and JMJD1B, in both immunoprecipitation and immunoblotting experiments (Supplemental Fig. S1B,C). Notably, all AETFC components could be coimmunoprecipitated with anti-JMJD1C in these cells (Fig. 1C) and AE-expressing SKNO-1 cells (Supplemental Fig. S1D). A subsequent analysis with Flag-tagged JMJD1C and HA-tagged AE proteins, expressed in and purified from insect cells, demonstrated a direct and stable interaction between these proteins (Fig. 1D; Supplemental Fig. S1E). In order to further document a direct interaction between AE and JMJD1C and provide information on which $\mathrm{AE}$ domain interacts with JMJD1C, High Five cells were coinfected with baculoviruses expressing HA-JMJD1C and different truncation variants of f-AE (Supplemental Fig. S1F), and lysates were immunoprecipitated with M2 agarose. The results clearly show that JMJD1C interacts with the $\mathrm{C}$ terminus of $\mathrm{AE}$, since AE9a, a spliced variant lacking the $\mathrm{C}$ terminus, showed no interaction (Supplemental Fig. S1G).

The coimmunoprecipitation of all AETFC components with anti-JMJD1C suggested that JMJD1C might interact with multiple AETFC subunits. Indeed, JMJD1C was coimmunoprecipitated (along with all AETFC subunits) by anti-HEB (Fig. 1E). More interestingly, an analysis with purified AE, AETFC, and combinations of AETFC subunits revealed that the interaction between $\mathrm{AE}$ and JMJD1C is more predominant in the context of the AEcontaining AETFC complex (Fig. 1F). These results encouraged us to examine whether other transcription factors in the AETFC complex could directly interact with JMJD1C and thereby effect stronger binding of JMJD1C to the AETFC complex relative to AE alone. In addition, HEB and E2A are ubiquitously expressed E-protein transcription factors that can act as homodimers or heterodimers with other tissue-restricted basic helixloop-helix proteins (Murre 2005) that include LYL1 (Visvader et al. 1991). In a further analysis, HA-HEB, HA-E2A, HA-HEB/f-LYL1, and HA-E2A/f-LYL1 were independently immobilized on HA agarose beads and then incubated with purified f-JMJD1C. Notably, JMJD1C 
Chen et al.

showed a direct interaction with the HEB homodimer (Fig. $1 G$, lane 6) and an even stronger interaction with the HEB/ LYL1 heterodimer (Fig. 1G, lane 4). This result suggests that multiple transcription factors in the AETFC complex, along with $\mathrm{AE}$, work together to interact with JMJD1C. This result is also consistent with an earlier report of the oncogenic potential of LYL1 in AML cells (Meng et al. 2005) and suggests that oncoprotein LYL1 dimerizes with HEB in the context of the AETFC complex to facilitate the interaction with JMJD1C.

\section{IMJD1C is necessary for survival of human acute myeloid leukemic cells}

To assess the role of JMJD1C in the survival of AEdependent cancer cells, we first depleted JMJD1C. The results, using two separate shRNAs, demonstrate that both Kasumi-1 cells (Fig. 2A) and SKNO-1 cells (Supplemental Figs. S2A, S9C,D) are very sensitive to depletion of JMJD1C, with cell numbers greatly reduced compared with control shRNA-treated cells. Similarly,
A

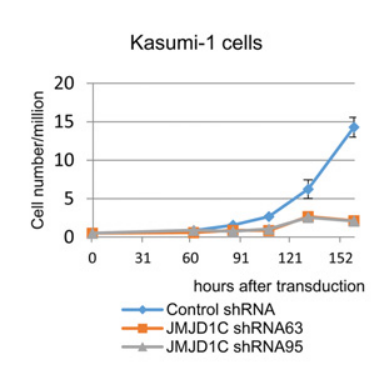

D

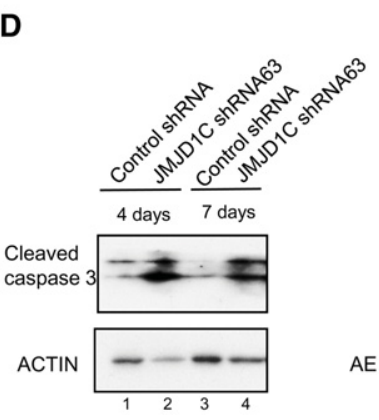

$\mathbf{G}$

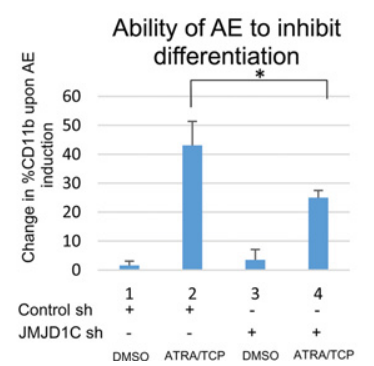

B

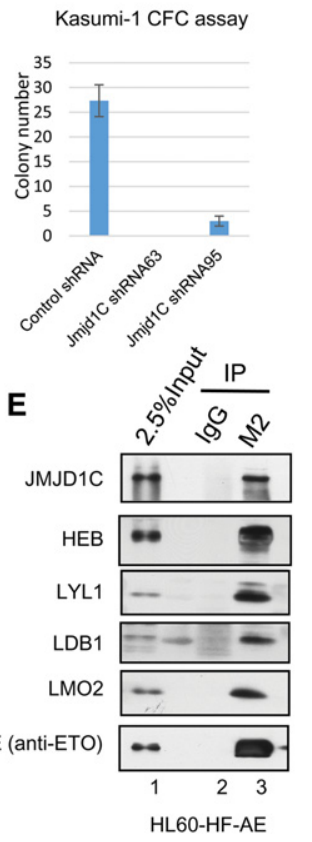

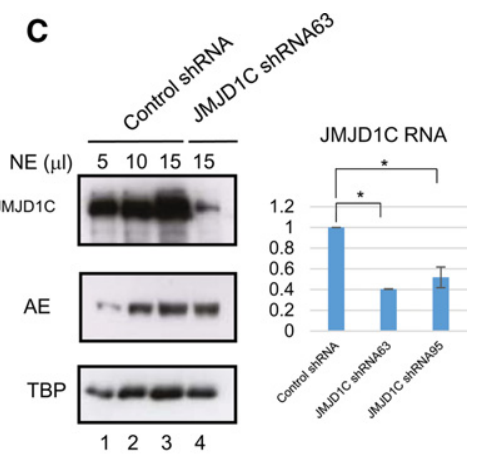

$\mathbf{F}$

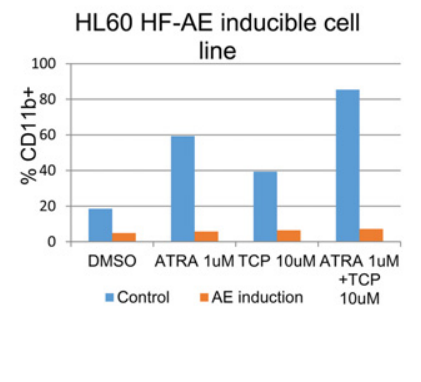

H

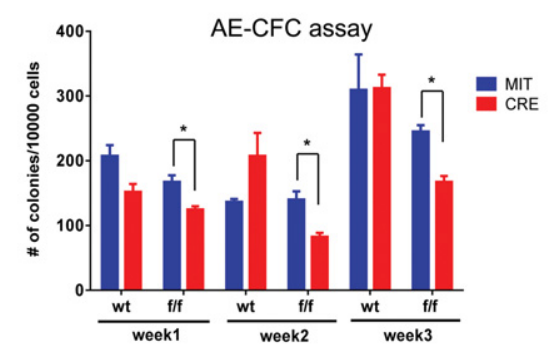

Figure 2. JMJD1C is required for Kasumi-1 cell growth and inhibition of differentiation by AE. $(A, B)$ Assessment of proliferation $(A)$ or colony formation ability $(B)$ of Kasumi-1 cells treated with either a control shRNA lentivirus or two separate JMJD1C shRNA lentiviruses. Error bars represent standard deviation (SD). Data in this figure are represented as mean \pm SD. $(C)$ Examination of JMJD1C protein $($ left $)$ and RNA (right) levels upon shRNA knockdown. $\left.{ }^{*}\right) P<0.05$. (D) Immunoblot showing increased cleaved caspase 3 upon JMJD1C knockdown. Actin blot served as a loading control. (E) Immunoprecipitation of HF-AE in HL60-HF-AE cells to confirm assembly of intact AETFC complex and JMJD1C association. Bound proteins were detected with antibodies to the proteins shown on the left. $(F)$ Quantification of CD11b-positive cell percentage from flow cytometry experiments indicating AE's ability to inhibit CD11b expression under different differentiation conditions in HL60-HF-AE cells. (G) Bar graph measuring the change of CD11b ${ }^{+}$percentage when AE is induced in HL60-HFAE cells with (lanes 3,4) or without (lanes 1,2) JMJD1C shRNA63. Quantification of three biological replicates of experiments is shown. $\left({ }^{*}\right) P<0.05$. $(H)$ Colony counts of AE transformed LIN $^{-} J$ Imjd1 $c^{f / f}$ bone marrow (BM) cells after transduction of CRE or MIT control viruses in methylcellulose. Results shown are from one wild-type and three $\mathrm{f} / \mathrm{f}$ mice. After each plating, colony numbers were counted per 10,000 cells plated. $\left(^{*}\right) P<0.05$. 
the colony-forming potential of Kasumi-1 cells was also reduced upon JMJD1C depletion (Fig. 2B), with the efficiency of JMJD1C knockdown assessed by both mRNA and protein levels (Fig. 2C). The reduced cell viability reflects increased apoptosis, as indicated by the increased level of cleaved Caspase 3 (Fig. 2D). However, no significant cell cycle alterations in Kasumi-1 cells were detected upon JMJD1C knockdown (Supplemental Fig. S2B). In summary, JMJD1C is required for survival of Kasumi-1 cells.

\section{JMJD1C plays a role in the AE-dependent inhibition of myeloid differentiation}

We next examined the role of AE in the inhibition of cell differentiation. We noted that HL-60 cells, which were derived from an FAB M2 acute myeloid leukemia patient (Dalton et al. 1988), can be induced to differentiate by ATRA in combination with an LSD1 inhibitor such as tranylcypromine (TCP) (Supplemental Fig. S2C, left column; Schenk et al. 2012). However, neither ATRA nor TCP, alone or in combination, could increase CD11b expression or reduce CD34 expression in Kasumi-1 cells (Supplemental Fig. S2C). Therefore, as an alternate approach, we established an HL-60 cell line (HL60-HF-AE cells) that can be induced to express $\mathrm{AE}$ in a doxycycline (Dox)-dependent manner (AE expression level is shown in Supplemental Fig. S1A). These cells grow at a similar rate without Dox induction (Supplemental Fig. S2D). More importantly, the exogenously expressed AE elicits assembly of the AETFC complex, which associates with JMJD1C (Fig. 2E). Cells were induced to differentiate by ATRA/TCP in the absence (control) or presence (AE induction) of Dox, and CD11b surface marker percentages were measured by flow cytometry. Notably, AE induction resulted in a dramatic reduction of CD11b expression under all differentiation conditions (Fig. 2F), confirming AE's ability to inhibit differentiation.

We next explored a potential role for JMJD1C in the AEdependent differentiation block in the inducible AE cell line. HL60-HF-AE cells were transduced with either control shRNA virus or JMJD1C shRNA63 virus and, after $2 \mathrm{~d}$, were treated with either solvent (DMSO) or ATRA/ TCP to induce differentiation. An analysis of CD11b expression revealed that the ability of $\mathrm{AE}$ to inhibit differentiation was greatly compromised by JMJD1C depletion (Supplemental Fig. S2E). Results from three independent cell lines are summarized in Figure 2G (cf. lanes 2 and 4). Depletion of JMJD1A or JMJD1B, two JMJD1C paralogs, did not affect AE's ability to inhibit differentiation (Supplemental Fig. S3A-D). These results suggest that JMJD1C, but not JMJD1A or JMJD1B, also plays a role in AE-dependent inhibition of cell differentiation.

\section{JMJD1C is important for the colony-forming activity of $A E$}

We next investigated whether the physical interaction between JMJD1C and AE plays an important role in AEdependent leukemia using a JMJD1C conditional knockout mouse. Since intact AE by itself does not generate leu- kemia in mice, we analyzed the leukemogenic potential of $\mathrm{AE}$ in a colony formation cell (CFC) assay using primary bone marrow-derived progenitor cells (Supplemental Fig. $\mathrm{S} 3 \mathrm{E})$. To this end, we isolated HSC-enriched cell populations from both wild-type and $\operatorname{mid} 1 c^{f / f}$ mice. Cells were first transduced with pMIGR-ha-AE-IRES-GFP. After GFP-positive cells were sorted, they were transduced with either MIT control or MIT-CRE viruses. Sorted cells were plated in Methocult supplemented with cytokines for colony formation. Although the same number of cells was plated for each sample, we observed a significant reduction in colony number with $J m j d 1 c^{f / f}$-CRE cells compared with $I m j d 1 c^{f / f}$-MIT cells after three rounds of plating (Fig. 2H). While the effect of Imjd1c deletion on AE-mediated colony formation is significant, it is also relatively mild compared with its effect on MLL-AF9mediated colony formation ( $\mathrm{N}$ Zhu and SA Armstrong, unpubl.). This mild effect, on the one hand, reflects some growth advantage of $J m j d 1 c$-undeleted cells, as indicated by genotype analyses showing the appearance of a Imjd1c-undeleted population at week 1 compared with day 2 after sorting (Supplemental Fig. S3F), and, on the other hand, is consistent with the JMJD1C requirement for AE-dependent colony formation. Notably, the reduction of CRE virus-treated cells compared with MIT virus-treated cells at week 1 is probably a result of CRE virus toxicity because no such reduction was observed at week 2 or 3 (Fig. 2H). These results suggest that JMJD1C is important for the colony-forming ability of progenitor cells driven by AE.

\section{JMJD1C and AE colocalize globally}

After establishing physical and functional connections between JMJD1C and AE, we set out to examine whether JMJD1C, which could potentially function as an AE coactivator, could be recruited to chromatin by AE and thereby play a role in the AE-directed transcriptional program. To this end, we performed ChIP-seq (chromatin immunoprecipitation [ChIP] combined with deep sequencing) analysis of JMJD1C in Kasumi-1 cells using our custom JMJD1C antibody. Our JMJD1C ChIP-seq results were compared with our earlier AE ChIP-seq results obtained with the ETO antibody (Sun et al. 2013). Well-established AE target genes such as LMO2, ID1, EGR1, and CDKN1A (also known as p21) are cobound by AE and JMJD1C in the same regions (Fig. 3A-D). Snapshots for two of the newly identified cobound genes, NOG and $P A D I 3$, are also shown (Fig. 3E,F) and further demonstrate the high quality of our ChIP-seq data and the colocalization of JMJD1C and AE.

An examination of the distribution of all JMJD1C-binding sites reveals that the JMJD1C peaks are significantly enriched toward promoter regions relative to the genomic DNA (12.9\% for JMJD1C vs. $1.1 \%$ for genomic DNA) (Supplemental Fig. S4A). To further demonstrate that JMJD1C colocalizes with AE in a genome-wide manner, we focused on flanking regions within $1 \mathrm{~kb}$ of the transcription start sites (TSSs) of all genes. First, presentation of the data in a Venn diagram shows a significant overlap 

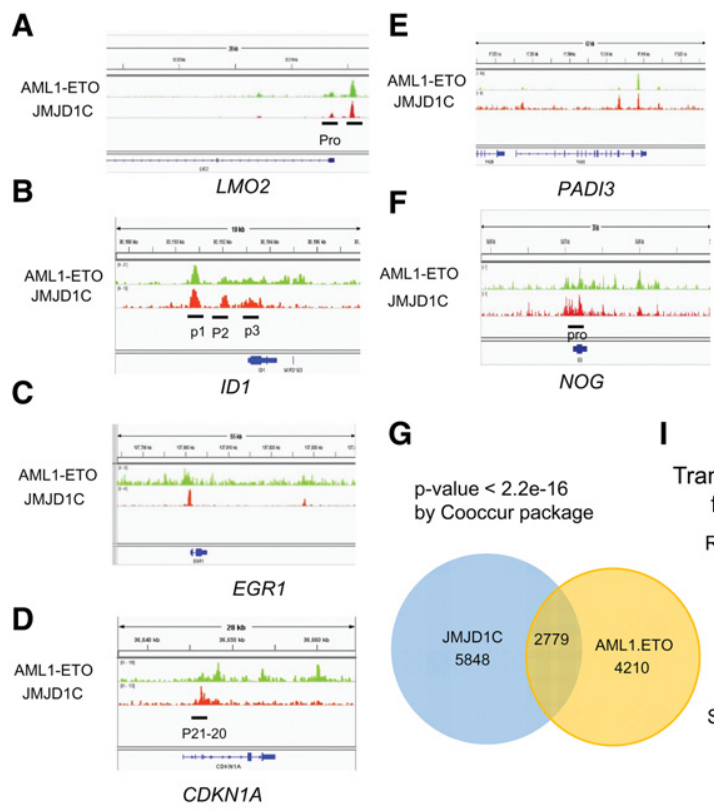

H
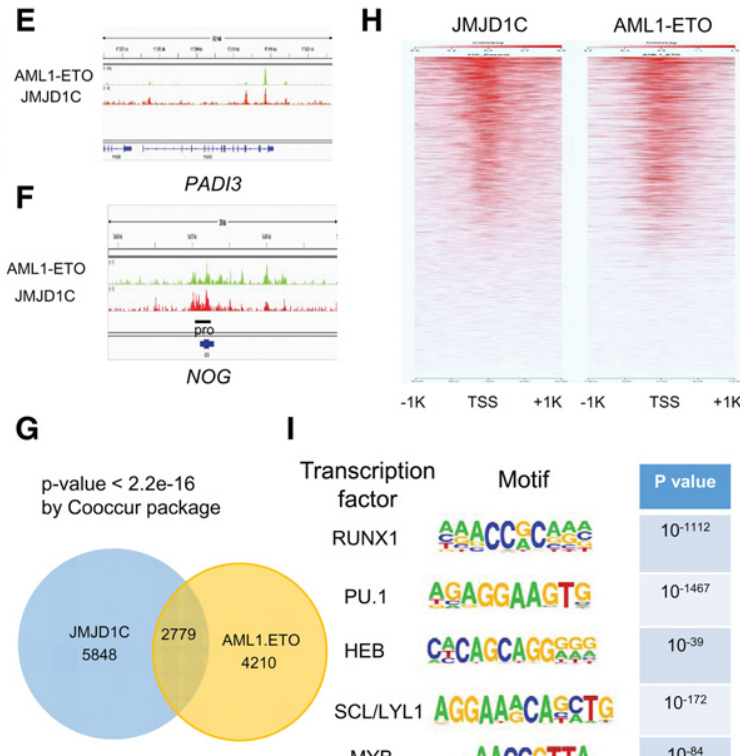

Transcrip
factor

RUNX1

PU.1

HEB

SCL/LYL1 AGGAAACAGCTG

MYB AACCGTTA

JMJD1C ChIP in Kasumi-1 cells

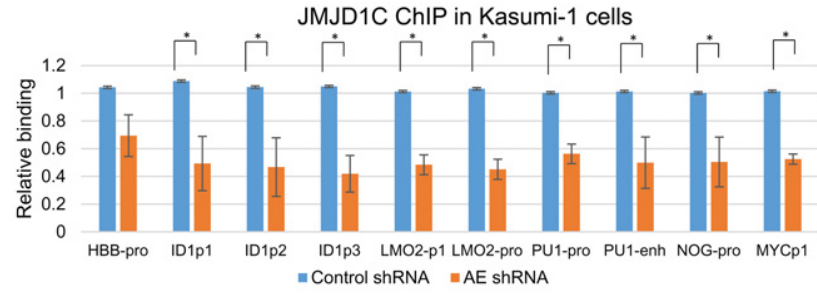

K

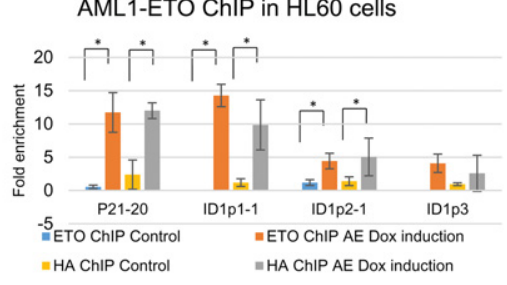

$\mathbf{L}$

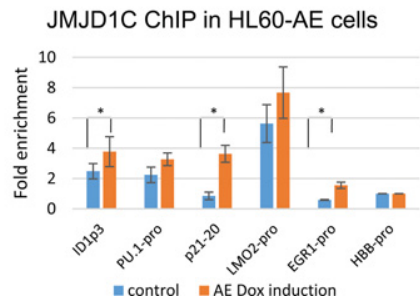

Figure 3. AE directly recruits its cofactor, JMJD1C, to target genes. $(A-F)$ ChIP-seq analyses of $\mathrm{AE}$ (green) and JMJD1C (red) on known AE-activated target genes LMO2, ID1, EGR1, and CDKN1A and newly identified target genes $P A D I 3$ and NOG. Track names are indicated at the left. Gene names are shown below each snapshot. Black bars indicate ChIP-PCR amplicons used in the quantitative PCR (qPCR) analyses in the following figure. $(G)$ Venn diagram depicting numbers of gene promoters (1-kb distance flanking transcription start sites [TSSs]) bound by JMJD1C alone (5848), by AE alone (4210), or jointly by both (2779). P-values assessing significance of the numbers of co-occupied genes are indicated above. $(H)$ Heat map of ChIP-seq reads for JMJD1C and AE rank-ordered from high to low JMJD1C occupancy centered on $\mathrm{a} \pm 1-\mathrm{kb}$ window around the TSSs of all genes. Color density reflects read enrichment; white indicates no enrichment. (I) Transcription factor-binding motifs enriched at JMJD1C-binding regions relative to genomic background, with associated $P$-values indicated at the right of each motif. $(J)$ ChIP-qPCR analyses of JMJD1C occupancy on target genes following control shRNA or AE shRNA treatment. Amplicon positions for peaks on LMO2 (LMO2-p1 and LMO2-pro), ID1 (ID1p1, ID1p2, and ID1p3), CDKN1A (p21-20), and NOG (NOG-pro) genes are schematically indicated as black bars in $A, B, D$, and $F$. ChIP amplicons for $H B B$ (HBB-pro), MYC (MYCp1), and SPI1 (PU.1-enh and PU.1pro) are indicated in Supplemental Figure S4E-G. Data in this figure are represented

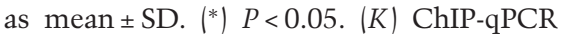
analyses of AE occupancy using either ETO antibody or HA antibody on target genes CDKN1A (p21) or ID1 in HL60-HF-AE cells.

Blue bars and yellow bars represent ChIP assays performed in HL60-HF-AE cells without Dox induction, whereas orange and gray bars represent ChIP assays performed in Dox-induced HL60-HF-AE cells. $\left(^{*}\right) P<0.05$. ( $L$ ) ChIP-qPCR analyses of JMJD1C occupancy on target genes upon AE induction in HL60-HF-AE cells. $\left({ }^{*}\right) P<0.05$.

of JMJD1C- and AE-bound promoters with a $P$-value of $<2.2 \times 10^{-16}$ (Fig. 3G). Moreover, the rank ordering of all TSS regions according to JMJD1C peak strength (Fig. 3H) or AE peak strength (Supplemental Fig. S4B) shows a positive correlation of JMJD1C and AE binding. In addition, a motif analysis using HOMER software showed significant enrichment of RUNX1-, HEB-, and SCL/LYL1-binding motifs in JMJD1C-bound regions obtained from JMJD1C ChIP-seq data (Supplemental Fig. 3I), consistent with our previous finding from AE ChIP-seq motif analysis that AE binds to DNA through both its Runt domain (DNA-binding domain of RUNX1) and its interaction with other AETFC components that also bind DNA directly (Sun et al. 2013). These results support our hypothesis that JMJD1C colocalizes with AE and likely the intact AETFC. We also identified motifs that correspond to binding sites for other hematopoietic transcription fac- tors that include PU.1/ETS, C/EBP, and AP-1 proteins. This finding is consistent with previously reported, likely dynamic, interactions of these transcription factors with AETFC components (Peterson and Zhang 2004; Wilson et al. 2010).

\section{IMJD1C is directly recruited by AE to target genes}

The direct physical interaction between AE and JMJD1C and their genome-wide colocalization support the idea that $\mathrm{AE}$, as a key transcription factor in Kasumi-1 cells, directly recruits JMJD1C to target genes. To further demonstrate that $\mathrm{AE}$ (or AETFC) is important for JMJD1C recruitment, we performed a JMJD1C ChIP-qPCR /quantitative PCR) assay following AE knockdown in Kasumi-1 and SKNO-1 cells. ChIP signals were normalized to control shRNA-treated cells. For the AE and JMJD1C 
coregulated target genes ID1, LMO2, and NOG, AE depletion resulted in notable decreases of JMJD1C occupancy in both Kasumi-1 (Fig. 3J) and SKNO-1 (Supplemental Fig. S4C,D) cells. These results strongly support our recruitment model.

To provide further evidence for AE-dependent JMJD1C recruitment, we again took advantage of the inducible HL60-HF-AE cell line. Both anti-HA and anti-ETO ChIP analyses revealed binding of HA-Flag-tagged AE to target genes following induction by Dox (Fig. 3K). Consistent with our previous results, AE induction also resulted in increased binding of JMJD1C on several target genes (Fig. 3L), with corresponding increases in RNA expression (Supplemental Fig. S5A) and with p21 being the most significant of the genes tested. Moreover, the increases in RNA expression were abrogated by depletion of JMJD1C (Supplemental Fig. S5B-D). Taken together, the data from both the loss-of-function and the gain-of-function studies strongly suggest that JMJD1C, as a binding partner of $\mathrm{AE}$, is directly recruited to $\mathrm{AE}$ target genes.

\section{JMJD1C regulates AE-activated genes in Kasumi-1 cells}

To investigate its role in regulating endogenous gene expression in Kasumi-1 cells, JMJD1C was depleted by shRNAs. We first examined effects on known AE-activated target genes that were identified in our earlier AE RNA sequencing (RNA-seq) analysis (Sun et al. 2013) and, as expected, showed that they were down-regulated at the RNA level by AE depletion (Fig. 4A, from p21 to STYK1). Depletion of JMJD1C using two shRNAs also resulted in a decrease in the RNA expression levels of these genes (Fig. 4B, from $p 21$ to STYK-1).

In order to examine how JMJD1C regulates target genes globally, we performed RNA-seq analyses of Kasumi-1 cells treated with either control shRNA or two JMJD1C shRNAs and with samples prepared in duplicate. We found that 517 and 327 genes were significantly downregulated in JMJD1C shRNA63-treated and JMJD1C shRNA95-treated cells, respectively, while 419 and 466 genes were significantly up-regulated in JMJD1C shRNA63-treated and JMJD1C shRNA95-treated cells, respectively. We then compared the RNA analyses with previously published (Sun et al. 2013) RNA-seq analyses of AE shRNA-treated Kasumi-1 cells. We picked several genes with FPKMs (fragments per kilobase per million mapped fragments) $>10$ in both JMJD1C shRNAtreated samples and AE shRNA-treated samples and confirmed their down-regulation by RNA-qPCR analysis in Kasumi-1 cells (Fig. 4A,B, from WASF1 to TSHZ3) and SKNO-1 cells (Supplemental Fig. S5E). We then set out to examine whether $\mathrm{AE}$ and JMJD1C regulate target genes coordinately in a genome-wide manner. We chose a cutoff of twofold change and $P$-value $<0.05$ for RNA-seq analysis from AE shRNA-treated cells. Venn diagrams show that, for both up-regulated and down-regulated gene categories, the RNA expression changes for both JMJD1C shRNA treatments are significant and consistent with the RNA expression changes following AE shRNA treatment (Fig. $4 C, D)$. Furthermore, box plots show that AE shRNA treat- ment significantly reduced expression of JMJD1C-activated genes $(P=0.000039)$ (Fig. 4E, left). AE and JMJD1C knockdowns also resulted in up-regulation of some genes. However, the overlap between these sets was only borderline significant $(P=0.042)$ (Fig. $4 \mathrm{E}$, right), suggesting that this is more likely to reflect secondary effects compared with the coordinately activated gene set. These results are consistent with the hypothesis that JMJD1C serves as a coactivator for $\mathrm{AE}$.

JMJD1C is necessary for the maintenance of low H3K9me1/2 levels on AE target genes in Kasumi-1 cells

Next, we aimed to understand the molecular mechanism of AE- and JMJD1C-dependent gene activation in leukemia cells. Although transcription cofactors can be recruited by different mechanisms to chromatin to exert their gene regulatory functions, one important mechanism is through binding to transcription factors that directly bind to DNA (Roeder 2005). In view of our demonstration of a direct interaction of JMJD1C with AE/AETFC, we postulated that, in Kasumi-1 cells, JMJD1C is directly recruited by $\mathrm{AE}$ to target genes in order to maintain $\mathrm{AE}$ target gene expression by removing the repressive H3K9me2 mark.

JMJD1C belongs to the same family as its paralog, JMJD1A, which has been shown to have histone H3K9me1/2 demethylase activity (Yamane et al. 2006). Although several studies have tried to assess the activity of JMJD1C, the conclusion is controversial (Kim et al. 2010; Brauchle et al. 2013; Watanabe et al. 2013; Sroczynska et al. 2014). Therefore, we first set out to address the discrepancy between different studies. In our in vitro studies with recombinant JMJD1C, we failed to observe any obvious demethylase activity on $\mathrm{H} 3 \mathrm{~K} 9 \mathrm{~m} 2$ peptide (data not shown), histone (Fig. 5A), or mononucleosome (Supplemental Fig. S6A) substrates but observed a weak demethylation of $\mathrm{H} 3 \mathrm{~K} 9 \mathrm{me} 1$ on a polynucleosome substrate (Fig. 5B). In view of the JMJD1C inactivity on histones and only weak activity on polynucleosomes as well as a precedent for other demethylases (Laurent et al. 2015), we considered the possibility that JMJD1C requires a cofactor for optimal demethylation activity. In support of this possibility, a NE fraction from $293 \mathrm{~T}$ cells potentiated the activity of JMJD1C, as evidenced by a clear reduction of H3K9me1 levels but not H3K4me1/2 levels on histone substrates (Fig. 5C).

Next, in order to further support our hypothesis of the function of JMJD1C as an H3K9 demethylase on target genes, we examined $\mathrm{H} 3 \mathrm{~K} 9 \mathrm{me} 2$ levels by ChIP assays in Kasumi-1 cells treated with JMJD1C or AE shRNAs. Although the coactivated genes examined were marked by varied amounts of $\mathrm{H} 3 \mathrm{~K} 9 \mathrm{me} 2$, most of the promoters showed increased levels of H3K9me2 following depletion of JMJD1C or AE (Fig. 5D; Supplemental Fig. S6B). Consistent with the increased levels of the repressive H3K9me2 mark and the decreased levels of gene expression (Fig. 4A,B) following AE and JMJD1C knockdown, the $\mathrm{H} 3 \mathrm{~K} 27$ acetylation mark $(\mathrm{H} 3 \mathrm{~K} 27 \mathrm{ac})$ that is associated 
Chen et al.

A

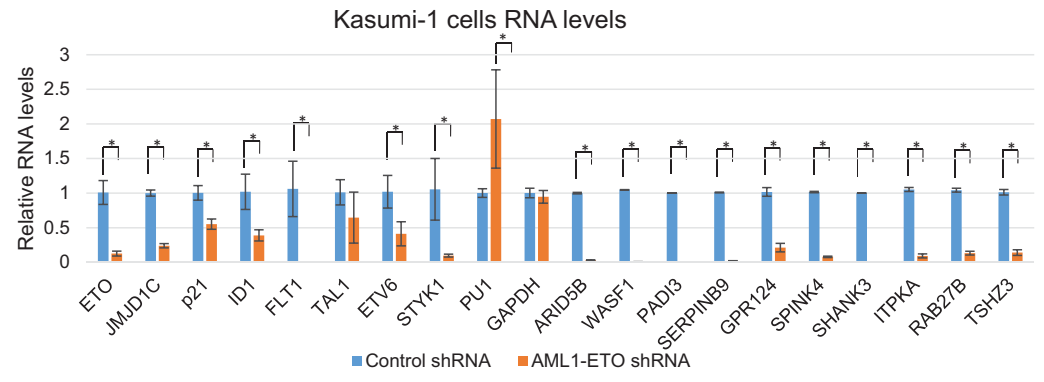

B

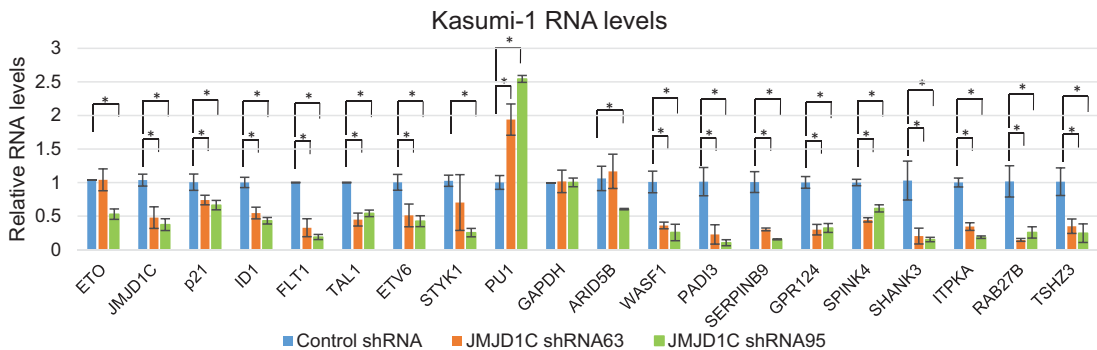

C

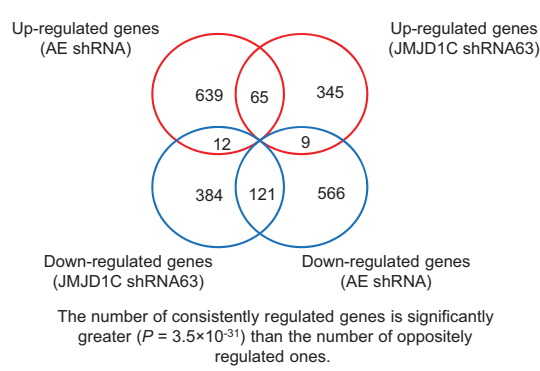

D

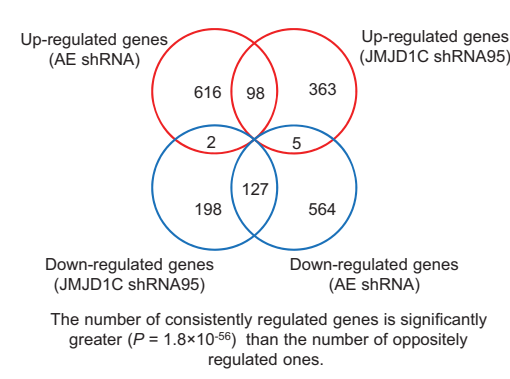

E

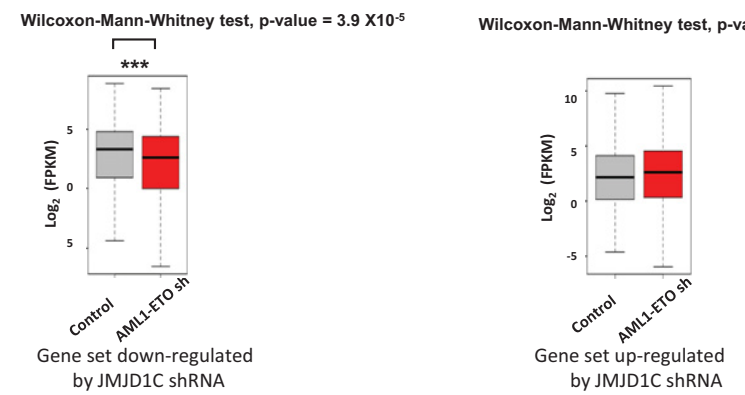

Figure 4. JMJD1C is required for expression of AE target genes. $(A, B)$ RT-qPCR analyses of RNA levels in Kasumi-1 cells treated with either control shRNA, AE shRNA $(A)$, or two separate JMJD1C shRNAs $(B)$. Control shRNA-treated samples were arbitrarily set as 1. Data in this figure are represented as mean $\pm \mathrm{SD} .\left({ }^{*}\right) P<0.05 .(C, D)$ Venn diagrams showing the numbers of genes differentially expressed with up-regulation (red) or down-regulation (blue) in Kasumi-1 cells treated with JMJD1C shRNA63 (C) or JMJD1C shRNA95 (D) and either control shRNA or AE shRNA. The number of consistently regulated genes is significantly greater $\left(P=3.5 \times 10^{-31}\right.$ or $P=1.8 \times 10^{-56}, \chi^{2}$ test) than the number of oppositely regulated ones. (E) Comparison of RNA expression levels [log(FPKM)] of control shRNA (Control) and AE shRNA-treated (AE) Kasumi-1 cells. (Left panel) Significantly down-regulated genes by JMJD1C knockdown with $q$-value $\leq 0.05$. (Right panel) Significantly up-regulated genes by JMJD1C knockdown with $q$-value $<0.05$. Control and AE at the bottom of each panel indicate the shRNAs used. $\left({ }^{* *}\right)$ Significance.

with activation was also reduced (Supplemental Fig. S7AC). In contrast, the H3K9me2 levels at the promoters of the $H B B$ gene, which is not expressed in Kasumi-1 cells, and the AE-repressed SPI1/PU.1 gene did not show significant changes (Fig. 5D; Supplemental Fig. S7D). In addition, $\mathrm{H} 3 \mathrm{~K} 9 \mathrm{me} 2$ levels of two representative genes were also shown to increase after AE or JMJD1C depletion in SKNO-1 cells (Supplemental Fig. S7E,F), consistent with our results in Kasumi-1 cells. Consistent with our in vitro demethylation assays, we also showed an increase of H3K9me1 levels on AE target genes upon JMJD1C depletion (Supplemental Fig. S7G-J).

To complement our knockdown experiments in Kasumi-1 cells, we used the AE inducible HL60-HF-AE cell line. We first compared ChIP-seq results for AE in Kasumi-1 (anti-ETO) and HL60-HF-AE (anti-HA) cells 
A

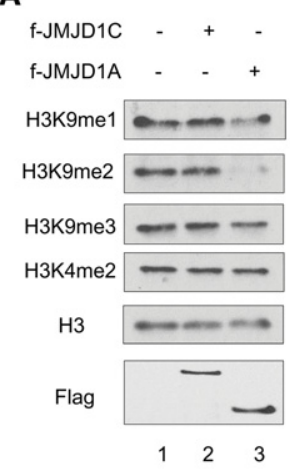

B

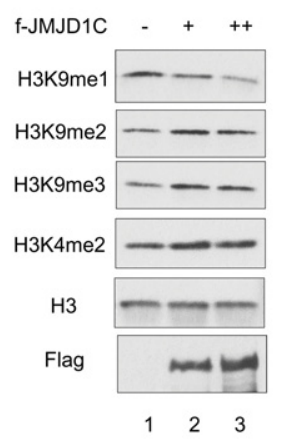

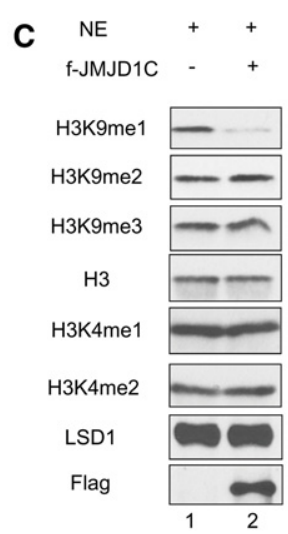

$\mathrm{H} 3 \mathrm{~K} 9 \mathrm{me} 2 / \mathrm{H} 3$

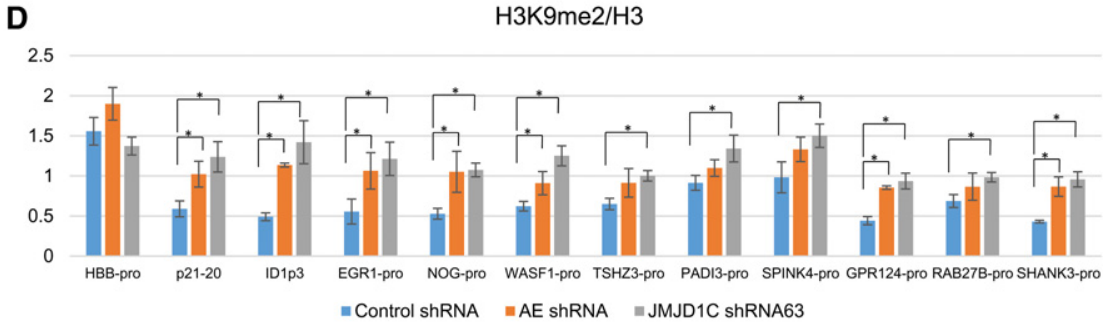

E
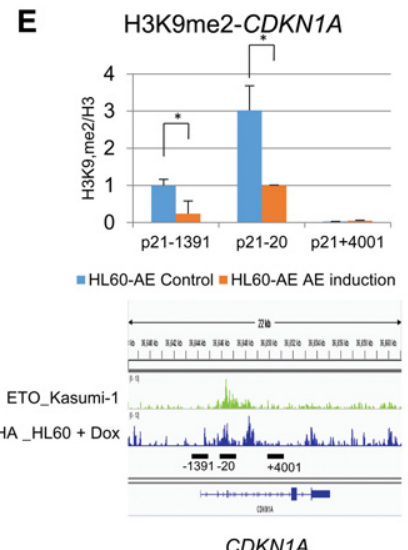

CDKN1A
$\mathbf{F}$

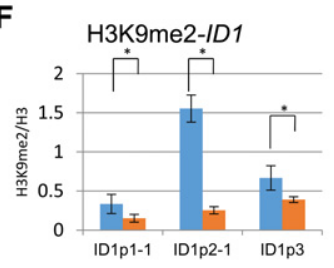

= HL60-AE Control $=$ HL60-AE AE induction

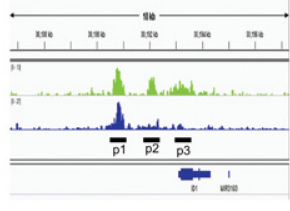

ID1
G

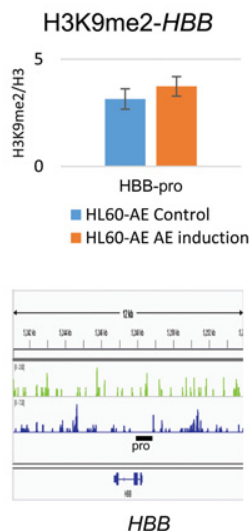

Figure 5. JMJD1C regulates $\mathrm{AE}$ target genes by maintaining low $\mathrm{H} 3 \mathrm{~K} 9 \mathrm{me} 2$ levels. (A) In vitro demethylation assays with purified f-JMJD1C (lane 2) and f-JMJD1A (lane 3) on histones. Antibodies are shown at the left. (B) In vitro demethylation assay with increasing amount of f-JMJD1C on polynucleosomes. $(C)$ In vitro demethylation assay with 293T NEs with (lane 2) or without (lane 1) purified f-JMJD1C. Antibodies are shown at the left. LSD1 is used as loading control for NEs. $(D)$ ChIP-qPCR analyses of $\mathrm{H} 3 \mathrm{~K} 9 \mathrm{me} 2$ levels on $\mathrm{AE}$ target genes in Kasumi-1 cells treated with either control shRNA (blue), AE shRNA (orange), or JMJD1C shRNA63 (gray). ChIP-PCR amplicons are described in Figure 3. Data in this figure are represented as mean $\pm \mathrm{SD}$. $\left.{ }^{*}\right) P<$ 0.05. (E-G, top) ChIP-qPCR analyses of H3K9me2 levels in HL60-HF-AE cells either without (blue) or with (orange) Dox induction on target genes $C D K N 1 A(p 21)$ and ID1 and on nontarget gene HBB. (Bottom) ChIP-seq analyses of AE occupancy in Kasumi-1 cells (green) using anti-ETO antibody or in HL60-HF-AE cells (blue) using anti-HA antibody. ChIP-PCR amplicons are indicated with black bars below the ChIP-seq tracks. The inactive $H B B$ gene is not occupied by either AE or JMJD1C and served as a negative control for $\mathrm{H} 3 \mathrm{~K} 9 \mathrm{me} 2$ level change. $\left.{ }^{*}\right) P<0.05$. after Dox induction. The results show that endogenous AE in Kasumi-1 cells and Dox-induced AE in HL60-HF$\mathrm{AE}$ cells bind to similar places on both CDKN1A and ID1 genes (Fig. 5E,F, bottom panels). We then designed three sets of primers for each gene to analyze different regions bound by $\mathrm{AE}$ in ChIP-qPCR assays (amplicons are indicated as black bars in Fig. 5E,F, bottom panels). We observed consistent reductions in $\mathrm{H} 3 \mathrm{~K} 9 \mathrm{me} 2$ levels on the CDKN1A and ID1 regions bound by AE upon Dox induction (Fig. 5E,F, top panels) but, similar to what was observed in Kasumi-1 cells, no change in the H3K9me2 levels on the $H B B$ promoter (Fig. 5G). Moreover, the depletion of JMJD1C with shRNA reversed the effect on H3K9me2 caused by AE induction, further confirming functional cooperation between AE and JMJD1C (Supplemental Fig. S8A). Examination of H3K18ac and H3K27ac levels showed increases of these marks only on TSSs (Supplemental Fig. S8B-E, PCR amplicons ID1p3 and p21-20). These results also suggest that $\mathrm{AE}$ directly recruits
JMJD1C to target genes and that this is accompanied by reductions in $\mathrm{H} 3 \mathrm{~K} 9 \mathrm{me} 2$ levels.

\section{JMJD1C is required for proliferation of multiple AML cell lines}

The above results have shown that JMJD1C directly interacts with $\mathrm{AE}$, is recruited to target genes activated by $\mathrm{AE}$, and is required for the maintenance of low $\mathrm{H} 3 \mathrm{~K} 9 \mathrm{me} 1 / 2$ levels at these genes in AE-dependent leukemia. An earlier discovery that JMJD1C is also involved in MLL-AF9 leukemia maintenance (Sroczynska et al. 2014) prompted us to examine whether JMJD1C is required in other leukemias. To this end, we examined the effect of JMJD1C depletion in various types of human AML cell lines (Supplemental Fig. S9A). First, RT-qPCR and immunoblot analyses showed that these cells express relatively high levels of JMJD1C RNA (Supplemental Fig. S9B) and similar levels of nuclear JMJD1C protein (Fig. 6A). Second, 


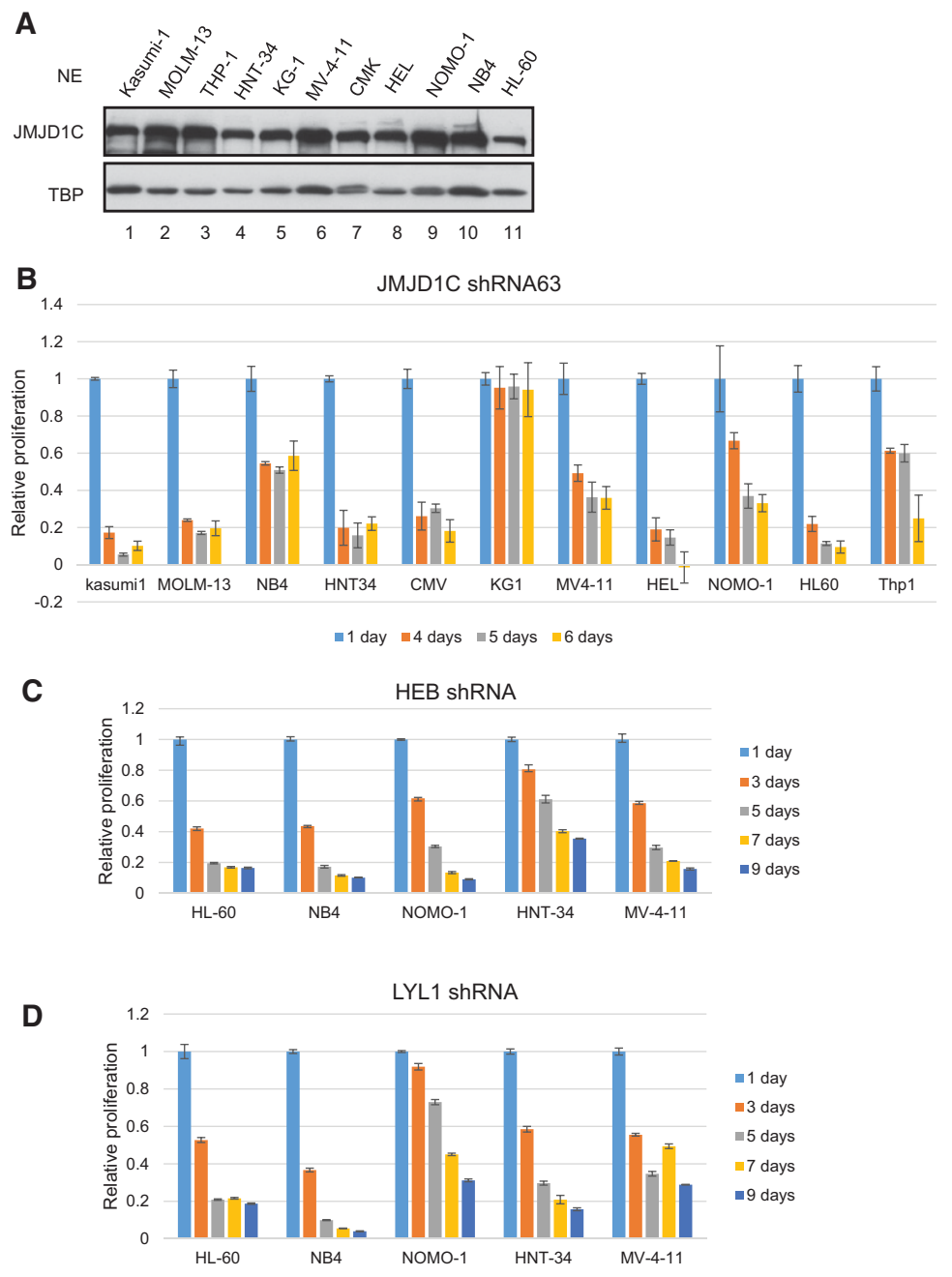

Figure 6. JMJD1C and HEB/LYL1 are essential for survival of a great variety of AML cells. (A) Immunoblot of JMJD1C protein levels in different leukemic cell NEs. TBP served as a loading control. $(B-D)$ Relative proliferation of human leukemia cell lines treated with JMJD1C shRNA63 $(B)$, HEB shRNA $(C)$, or LYL1 shRNA $(D)$. Proliferation was measured by cell viability from $1 \mathrm{~d}$ after puromycin selection. Cell numbers obtained from shRNA-treated cells were set to 1. Data in these figures are represented as mean $\pm \mathrm{SD}$. depletion of JMJD1C compromised the growth of all tested AML cell lines except KG-1 (Fig. 6B; Supplemental Fig. S9C,D). These results suggest that JMJD1C, a commonly expressed protein in AML cell lines, is required for maintenance of multiple types of leukemia that result from different mutations.

IMJD1C associates with key transcription factors or transcription factor complexes in different leukemia cell lines

The surprising fact that JMJD1C is apparently required for multiple types of AML encouraged us to look for a molecular mechanism that supports a common consequence of JMJD1C depletion from distinct cell types. Our current evidence supports a model in which AETFC recruits JMJD1C to its target genes in Kasumi-1 cells to regulate transcription. In this regard, our finding that JMJD1C in Kasumi-1 cells not only interacts with AE alone but also associates even more strongly with other components of the AETFC complex implies that JMJD1C works through this key multicomponent transcription factor complex (Fig. 1C). This observation and our broader functional data for JMJD1C led us to first hypothesize that shared transcription factors in different AML cells may cooperate with key leukemogenic transcription factors to recruit JMJD1C and, second, search for JMJD1C interactions with key transcription factors in other AML cell lines.

Because LYL1 and HEB are highly expressed in AMLs, important for AE-driven leukemia (Sun et al. 2013), and interact directly with JMJD1C (Fig. 1G), we postulated that, as observed in Kasumi-1 cells, they might play a role in recruiting JMJD1C in other AML cell lines for which JMJD1C is required for cell growth. To this end, we first demonstrated that HEB and LYL1 are required for the growth of several non-AE AML cells that are sensitive to JMJD1C depletion, including HL60 and NB4 as well as MLL fusion cell lines (Fig. 6C,D). In addition, similar to observations with AE-dependent cell lines, we consistently detected an association of HEB and LYL1 with JMJD1C in these cells (Fig. 7A) but not KG-1 cells (Supplemental Fig. S9E). Interestingly, we observed a very weak association of MYB with JMJD1C in Kasumi-1 cells but not two other tested cell lines (HL-60 and NB4) (Supplemental Fig. S9F). These results are consistent with the observed enrichment of the MYB-binding motif in the JMJD1C ChIP-seq analysis in Kasumi-1 cells (Fig. 3I) and, 

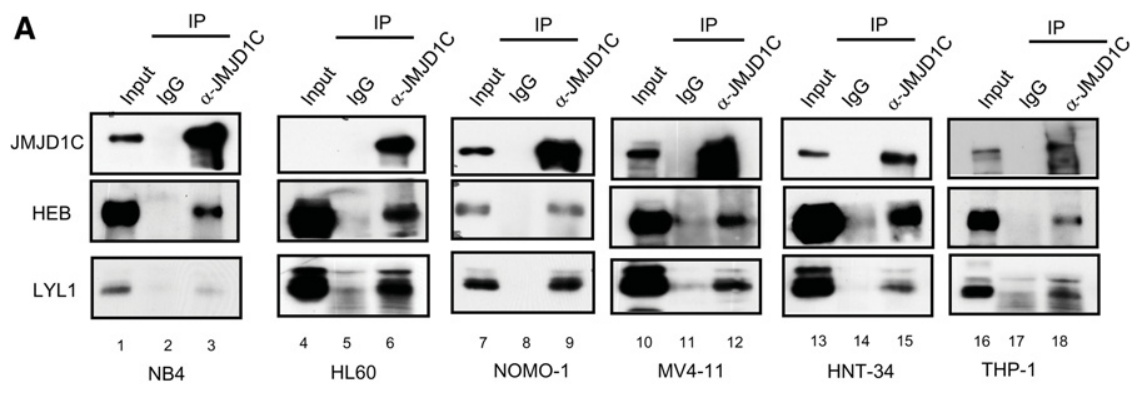

B
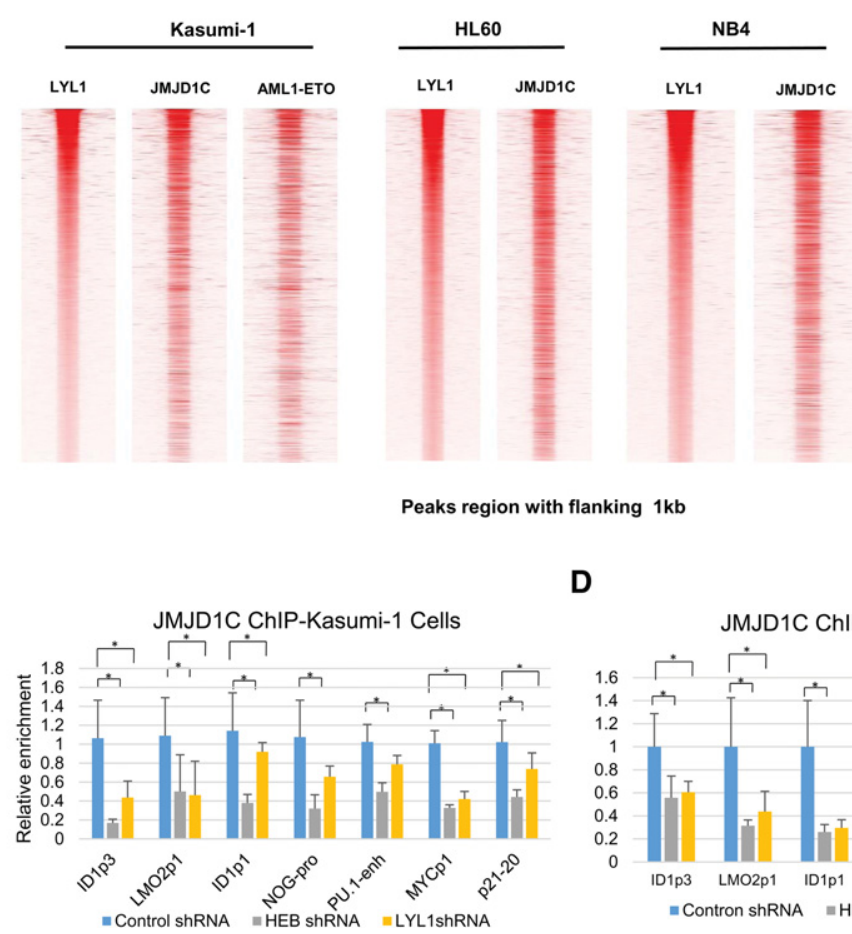

$1 \mathrm{~kb}$

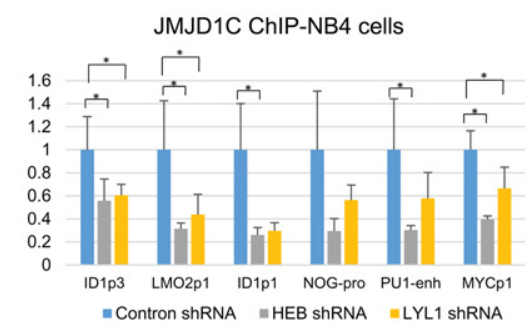

Figure 7. HEB/LYL1 are important for JMJD1C recruitment in AMLs. (A) Immunoprecipitation of JMJD1C from different AML cells (indicated below each panel) to confirm HEB and LYL1 association. Bound proteins were detected with antibodies to proteins shown at the left. (B) Heat maps of ChIP-seq reads for JMJD1C, LYL, and AE (only for Kasumi-1 cells) rank-ordered from high to low LYL1 occupancy centered on $\mathrm{a} \pm 1-\mathrm{kb}$ window around the peaks of all binding regions of LYL1. Color density reflects read enrichment; white indicates no enrichment. $(C, D)$ HEB and LYL1 are important for JMJD1C occupancy on target genes in Kasumi-1 $(C)$ and NB4 $(D)$ cells. ChIP-qPCR analyses of JMJD1C occupancy on target genes following control shRNA, HEB shRNA, or LYL1 shRNA treatment. Data are represented as mean $\pm \mathrm{SD} .\left({ }^{*}\right) P<$ 0.05 .
D

C

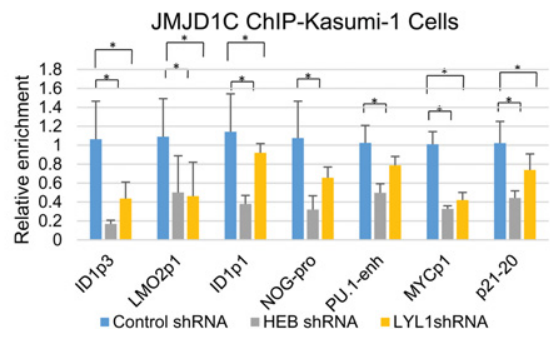

more generally, show specificity of HEB and LYL1 as shared transcription factors in various AML cells to recruit JMJD1C.

In view of the physical interaction between HEB/LYL1 and JMJD1C and their requirement for leukemic cell growth, we proposed that HEB and LYL are important for JMJD1C chromatin association. To test this idea, we performed ChIP-seq analyses of JMJD1C and LYL1 in several AML cell lines that included Kasumi-1 cells and three non-AE cell lines (HL60, NB4, and THP1). In order to demonstrate genome-wide colocalization of LYL1 and JMJD1C, we generated heat maps that were rank-ordered according to LYL1-binding strength. First, and as expected, both JMJD1C and AE showed strong correlations with LYL1 peaks in Kasumi-1 cells, consistent with residency of LYL1 and AE in the same protein complex. More importantly, and as anticipated, genome-wide analyses also showed a strong correlation between JMJD1Cand LYL1-binding regions in the HL60, NB4, and THP1 cells (Fig. 7B).

In further support of the idea that HEB and LYL1 are critical for JMJD1C recruitment, shRNA-mediated depletion of HEB or LYL1 in Kasumi-1, NB4, and HL60 cells in- deed reduced JMJD1C recruitment (monitored by ChIPqPCR) to JMJD1C target genes (Fig. 7C,D; Supplemental Fig. S9G). These observations suggest that, in AML cells, these E-box-binding proteins may work through formation of corresponding transcription factor complexes with distinct key transcription factors and that these different complexes may directly recruit JMJD1C to specific target genes for cell type-specific transcriptional regulation. Together with our results for Kasumi-1 cells, our protein-protein interaction and ChIP-seq analyses strongly support our hypothesis that the aberrantly expressed leukemic fusion proteins work together with more broadly expressed transcription factors, which are important for leukemia, to recruit JMJD1C to their target genes to regulate gene expression.

\section{Discussion}

In this study, we identified the histone demethylase JMJD1C as a factor that physically associates with AE and functions as a coactivator to maintain the AE-dependent leukemic gene expression program. These results suggest a model in which the AE-containing AETFC 
complex (Sun et al. 2013) recruits JMJD1C to AE target genes to counter the accumulation of $\mathrm{H} 3 \mathrm{~K} 9$ methylation, a silencing mark known to epigenetically silence stem cell genes during myeloid differentiation (Chen et al. 2012). Intriguingly, we found that JMJD1C interacts with not only AE and other components in the AETFC complex but also several other leukemogenic transcription factors that drive AML (see below), suggesting that the requirement for JMJD1C is a general phenomenon in myeloid leukemias. This finding indicates the potential of JMJD1C as a therapeutic target for treatment of AML patients with multiple cytogenetic backgrounds.

\section{IMJD1C as a coactivator for AE-dependent} transcriptional regulation in Kasumi-1 cells

Our rigorous biochemical results with purified proteins confirmed our coimmunoprecipitation-mass spectrometry results and established a direct physical interaction between AETFC and JMJD1C. The significance of this interaction was borne out by JMJD1C knockdown and RNA expression analyses, which demonstrated that JMJD1C and $\mathrm{AE}$ coregulate a large set of AE-activated genes in Kasumi-1 cells. Consistent with the physical interaction between AE and JMJD1C, genomic (ChIP-seq) analyses showed extensive colocalization of JMJD1C with AE near TSSs. Additional studies showed that JMJD1C recruitment is impaired upon AE knockdown in Kasumi-1 cells, whereas JMJD1C is recruited to new AE-bound loci when AE is ectopically expressed in HL-60 cells, strongly supporting a mechanism involving direct recruitment by AE. Notable in this regard is our demonstration that the JMJD1C interaction with $\mathrm{AE}$ is through the C-terminal region that is lacking in AE9a. AE9a is a spliced AE isoform that, in contrast to fulllength $\mathrm{AE}$, promotes leukemia in a mouse model without secondary mutations (Yan et al. 2006). Therefore, our demonstration of a novel biologically important interaction of the $\mathrm{AE} \mathrm{C}$ terminus provides an additional indication of the mechanistic complexity underlying natural AE-dependent leukemia. Apart from earlier reports of HDAC interactions with the C-terminal region of AE (Gelmetti et al. 1998; Lutterbach et al. 1998; Wang et al. 1998), a recent report from the Zhang group (DeKelver et al. 2013) also demonstrated important contributions of $\mathrm{AE}$ interactions, mediated by the C-terminal NHR4 domain, to leukemogenesis. Altogether, past and current results suggest the existence of AE C-terminal domain-binding partners (coactivators and corepressors) with opposing functions and further underscore the importance of developing leukemia models for the full-length AE protein.

The identification of consensus motifs for myeloid transcription factors within AE-and JMJD1C-binding peaks further supports a model for JMJD1C function in AML that involves recruitment by transcription factors, since cofactors, especially histone-modifying factors such as JMJD1C, generally lack site-specific DNA-binding capacity and thus require interactions with transcription factors for localization to target genes. The Runxl motif was identified as one with the lowest $P$-value, suggesting that AE containing the RUNT domain of AML1/ RUNX1 is a primary determinant of JMJD1C binding in Kasumi-1 cells. We also identified motifs corresponding to HEB and SCL/LYL1, integral members of AETFC, indicating that JMJD1C may also bind to genomic regions together with the intact AETFC complex. Consistent with this suggestion, biochemical experiments showed that JMJD1C can bind HEB/LYL1 as well as AE and that it binds more strongly to AETFC than to AE alone.

In agreement with the physical interaction and genomic localization results, our global RNA expression analysis showed that JMJD1C and AE regulate target gene expression coordinately. In addition, and consistent with JMJD1C being an AE coactivator, our analysis also confirmed a greater coregulation in the AE-activated gene group than in the AE-repressed gene group.

\section{JMJD1C is required for survival of AML cells}

While our work on JMJD1C was initiated on the basis of our observation that JMJD1C interacts with AE, we also found that all AML subtype cell lines tested had a striking dependence on JMJD1C. Consistent with our findings, a requirement for JMJD1C in MLL fusion leukemia and several cell lines, including U937 and K-562, has recently been shown by others (Sroczynska et al. 2014). In our AE leukemic model, we observed that JMJD1C is essential for extended colony-forming ability of mouse progenitor cells transduced with AE-expressing virus, which reflects an essential role of JMJD1C in AE-dependent proliferation. Mechanisms that have been proposed for the function of $\mathrm{AE}$ in blocking differentiation include inhibition of the expression of C/EBPa /a bZIP transcription factor critical for granulocytic differentiation) (Pabst et al. 2001) and inhibition of the transcription activity of PU.1 through a direct physical interaction (Vangala et al. 2003). In relation to a role for JMJD1C in the inhibition of AE-dependent differentiation, our RNA-seq analyses of JMJD1C- and AE-depleted Kasumi-1 cells indicated significant cooperativity in gene regulation. One interesting gene jointly targeted by $\mathrm{AE}$ and JMJD1C is NOG, which encodes a signaling molecule that inhibits the activity of BMPs (Groppe et al. 2002). As a preliminary analysis has revealed cell death following NOG depletion in Kasumi1 cells, it will be interesting to determine whether inhibition of BMP signaling is involved in blocking the differentiation of certain AMLs.

Identification of key JMJD1C target genes is also of great importance for an understanding of its requirement for the survival of AML cells. Based on a gene ontology (GO) analysis, JMJD1C-activated genes in Kasumi-1 cells were enriched in genes encoding factors that enhance proliferation, transmembrane proteins involved in tyrosine kinase signaling pathways, and proteins involved in cell differentiation. Whether these genes are specific to the AE-regulated transcription program or are relevant to JMJD1C's more general role in AML survival remains to be determined through more thorough studies, as does the identification of direct target genes. The identification of 
overlapping JMJD1C target genes in different AMLs could reveal common pathways that are critical for leukemic cells, whereas the identification of unique target genes in these AML cells could reveal programs that are specific to individual leukemic programs and dictated by specific transcription factors.

\section{JMJD1C as a cofactor for multiple AML cells}

Tissue diversity is usually defined by expression of a combination of tissue-specific or cell type-specific transcription factors, while cofactors such as chromatin-modifying enzymes and remodeling complexes tend to be expressed in a much more ubiquitous manner. Like other transcriptional cofactors, JMJD1C is expressed at similar levels in the different human leukemic cells examined in this study. However, consistent with well-established concepts, analyses of transcription factors have revealed much more diverse expression patterns in the various leukemias. For example, transcription factor LYL1, which is a core component of AETFC in Kasumi-1 cells, is expressed at high levels in AML cell lines and is barely detectable in pro-B leukemia 697 cells (data not shown). This observation is consistent with previous indications that LYL1 is expressed at higher levels in the majority of AML or myelodysplastic syndrome cases relative to normal bone marrow (Meng et al. 2005).

One key question is why JMJD1C is required in multiple AML cell types. Our analyses indicate that JMJD1C functions as a transcriptional coactivator that is recruited by different transcription factors. How might JMJD1C interact with such structurally diverse transcription factors? One possibility is that some factors in the transcription complex nucleated by AE in Kasumi-1 cells account for the recruitment of JMJD1C to key loci in other AML cell types. Thus, although JMJD1C interacts directly with AE, it interacts much more strongly with the AETFC complex in Kasumi-1 cells (Fig. 1F). In fact, several studies, including our recent AE study (Sun et al. 2013), have shown that many hematopoietic transcription factors act cooperatively to regulate transcription (Tripic et al. 2009; Wilson et al. 2010). Among these factors, LDB1 and LMO2 function as scaffolding proteins to assemble large protein complexes consisting of specific E-box-binding proteins (LYL1, HEB, or E2A) or GATA proteins, etc. (Wadman et al. 1997; Lecuyer and Hoang 2004; El Omari et al. 2013). Indeed, our results show not only the importance of HEB/LYL1 in AML cell survival but also a direct interaction of these factors with JMJD1C that could contribute to JMJD1C recruitment. This observation suggests that, in diverse AML cells, these E-box-binding proteins may work with distinct leukemic transcription factors to recruit JMJD1C to specific target genes for cell type-specific transcriptional regulation. This property of JMJD1C is reminiscent of that of the large coactivator p300, which interacts directly with several transcription factors (including AE and HEB) in the AETFC complex. This ability of key transcription cofactors with multiple protein interaction domains to interact with leulemogenic transcription factor complexes enables them to be more efficiently recruited to important target genes to maintain cell identity.

Another important question is whether HEB and LYL1 are the only shared transcription factors that JMJD1C and/ or p300 (also required for leukemic cell maintenance) rely on for recruitment to key target genes. Blood cell identity is controlled by combined functions of multiple transcription factors (Wilson et al. 2010; Martens et al. 2012), as reflected by their overlapping genomic localization and direct protein-protein interactions. In fact, key transcription factors important for blood progenitor/stem cells have been shown to be critical for several leukemias. These include the requirement of a wild-type copy of RUNX1 for AE-dependent leukemia (Ben-Ami et al. 2013) and the requirement of PU.1 and C/EBPa for MLL fusion-related leukemia (Ohlsson et al. 2014; Zhou et al. 2014). Understanding the shared mechanism by which JMJD1C is recruited to chromatin in different types of leukemia is of great importance in that a common targeted therapy could be identified through such a universal mechanism. One way to identify such a mechanism is by examination of shared DNA-binding motifs to which JMJD1C preferentially binds in different leukemias. Another possibility is through biochemistry/coimmunoprecipitation analyses of JMJD1C in different AML cells to identify common interacting transcription factors.

\section{Demethylase activity of JMJD1C}

JMJD1C resides within a common family that includes JMJD1A $(K D M 3 A)$ and JMJD1B $(K D M 3 B)$, both of which show in vitro demethylation activities on $\mathrm{H} 3 \mathrm{~K} 9 \mathrm{me} 1$ and H3K9md2 (Yamane et al. 2006; Brauchle et al. 2013). Mouse JMJD1C also has been reported to function as a bona fide histone demethylase both in vitro and in cells (Kim et al. 2010). Consistent with these results, we demonstrated an $\mathrm{H} 3 \mathrm{~K} 9 \mathrm{me} 1$ demethylase activity for human JMJD1C in vitro and further showed in both loss-of-function and gain-of-function analyses that JMJD1C intracellular expression is critical for the maintenance of low H3K9me1/2 levels on its target genes. In agreement with other studies of the cellular function of mouse and human JMJD1C (Brauchle et al. 2013; Sroczynska et al. 2014) and likely reflecting gene-selective functions of JMJD1C or redundancy among family members, we did not detect global changes of the H3K9me2 level following knockdown of AE or JMJD1C in Kasumi-1 cells (data not shown). On the other hand, our recombinant human JMJD1C failed to show an obvious demethylase activity on $\mathrm{H} 3 \mathrm{~K} 9 \mathrm{me} 2$ peptide, histone, and mononucleosome substrates, in contrast to the results of Kim et al. (2010), but did exhibit a weak H3K9me1 demethylase activity on a polynucleosome substrate. These results raised the possibility that JMJD1C, like certain other histone demethylases, may have a low substrate affinity that contributes to its inactivity at the low concentration of purified protein assayed in vitro and/or may require a cofactor for optimal activity. As an example, a neuronal isoform of LSD1 (LSD1-8a) that has gene-specific H3K9me2 demethylase 
activity in cells requires the presence of a cofactor for in vitro activity and, when depleted in cells, has little effect on global H3K9me2 levels (Laurent et al. 2015). Notably, and consistent with the results of the LSD1-8a study, a NE fraction that showed no activity on its own was found to stimulate significantly the H3K9 demethylation activity of JMJD1C. Although the LSD1-8a cofactor has been identified as SVIL, the JMJD1C cofactors remains to be identified and presents a future problem of great interest for both mechanistic and inhibitor-screening studies for JMJD1C.

\section{IMJD1C as a potential therapeutic target}

Our results indicate that depletion of JMJD1C leads to growth impairment of a variety of leukemic cell types that includes cells expressing AE. Another recent study reported similar effects of JMJD1C depletion on MLL rearranged leukemic cells along with a few cell lines, including U-937 and K-562, and a lack of noticeable effects on normal hematopoietic cells (Sroczynska et al. 2014). Our combined results suggest that JMJD1C is a potentially relevant drug target for multiple leukemias. In this regard, small molecule inhibitors have been successfully developed for jmjC domain-containing demethylases. For example, GSK-J1 and GSK-J5 selectively inhibit KDM6 family members that modulate $\mathrm{H} 3 \mathrm{~K} 27 \mathrm{me} 3$ levels to regulate the proinflammatory macrophage response (Kruidenier et al. 2012). Another example is methylstat, which preferentially inhibits KDM4A (lower IC50) relative to other JmjC domain-containing demethylases (Luo et al. 2011). Notably, the establishment of JMJD1C demethylase activity in vitro will facilitate screens for selective demethylase inhibitors. Taken together, our findings implicate JMJD1C as a crucial gene in leukemia and qualify it as a potential therapeutic target in leukemia subtypes spanning a range of lineages and cytogenetic states.

\section{Materials and methods}

\section{Protein purification and mass spectrometry}

Kasumi-1 cells were grown in 12-L spinner flasks (BellCo), and the NE was prepared by our standard high-salt extraction method (Dignam et al. 1983). Complex purification was performed in buffer BC150 (20 mM HEPES at pH 7.9, 150 mM KCl, 0.2 mM EDTA, $10 \%$ glycerol) plus $0.1 \%$ NP-40. Eluted proteins were separated by SDS-PAGE, and visible bands on the gel were sliced separately into 10 bands and subjected to liquid chromatography coupled with tandem mass spectrometry (LC-MS/MS) analysis.

\section{Cell culture and treatment}

Human leukemic cell lines were cultured in RMPI-1640 medium (Life Technologies) with 10\% fetal bovine serum (Life Technologies). For differentiation analyses, $1 \mu \mathrm{M}$ ATRA and/or $10 \mu \mathrm{M}$ TCP (in DMSO) was added to the cultures. Forty-eight hours later, cells were labeled with CD11b-PE (BD Biosciences) or CD34FITC (BD Biosciences) antibodies and PI (Biolegend) and analyzed by Accuri C6 flow cytometer (BD Biosciences). Apoptotic cells were analyzed by labeling with Annexin V and PI (BioLegend) 4 $\mathrm{d}$ after shRNA virus infection.

\section{In vitro binding and immunoprecipitation studies}

The Bac-to-Bac baculovirus expression system (Invitrogen) was used to generate recombinant f-JMJD1C, its variants, and recombinant AETFC components. Reconstituted protein and protein complexes were purified from High Five insect cells coinfected with different combinations of baculoviruses.

\section{ChIP and ChIP-seq analysis}

ChIP and ChIP-seq analysis were performed as described in the Supplemental Material.

\section{shRNA knockdown}

The shRNA against AE was designed to target the fusion site (Heidenreich et al. 2003). Other lentiviral shRNA sets were purchased from Sigma (sequences are shown in the Supplemental Material). Virus preparation and cell infection were performed according to the manufacturer's protocol. Knockdown efficiencies were analyzed by RT-qPCR and/or immunoblot at $4 \mathrm{~d}$ after transduction.

\section{RNA-seq}

Kasumi-1 cells with JMJD1C depletion were harvested at $4 \mathrm{~d}$ after viral transduction, and RNA was extracted with RNeasy Plus kit (Qiagen). RNA-seq library preparations were done using established Illumina methods for mRNA-seq (part no. RS-1222001). Libraries were generated using the TruSeq RNA sample preparation kit (Illumina) and sequenced with an Illumina HiSeq 2500.

\section{Cell transfection and coimmunoprecipitation}

293T cells were transfected using Lipofectamine 2000 (Invitrogen). For coimmunoprecipitation experiments, NEs were made as described (Dignam et al. 1983) and diluted with BC0/0.2\% Triton X-100 buffer. After clearance of protein precipitants, NEs were incubated with antibody-conjugated protein A agarose beads. Beads were then washed four times with BC150/0.1\% NP40, and proteins were analyzed by immunoblot.

\section{Bone marrow transduction and colony formation assay and analysis}

Bone marrow transduction and colony formation assay and analysis were performed as described in the Supplemental Material.

\section{Accession code}

The data for ChIP-seq and RNA-seq analyses can be found at Gene Expression Omnibus GSE63486.

\section{Competing interest statement}

Y.S. is a cofounder of Constellation Pharmaceuticals, Inc., and is a member of its scientific advisory board.

\section{Acknowledgments}

We thank Dr. Yi Zhang for the pCDNA3-f-JHDM2A plasmid, Dr. Hanez Ruffner for pcL-MSCV-JMJD1C and pcL-MSCV-Jmjd1B plasmids, Dr. Xiaojian Sun for pFastbacl vectors expressing 
AETFC components, Dr. Dong-Er Zhang for Migr-ha-AE constructs, Dr. Chris Vakoc for AML cell lines, Roeder laboratory members for helpful discussions, the Proteomics core facility at the Rockefeller University for mass spectrometry, and the Epigenomics core at Cornell Weill Medical College for ChIP-seq and RNA-seq library generation and sequencing. This work was supported by grants from the National Institutes of Health (CA163086), the Starr Cancer Consortium (I5-A555), and the Leukemia and Lymphoma Society (7132-08) to R.G.R.; grants from the National Institutes of Health (CA176745 and CA66996) to S.A.A.; a grant from the National Institutes of Health (R01 CA118487) to Y.S.; a career development fellowship (5666-13) from the Leukemia and Lymphoma Society to M.C.; and a grant from the National Institutes of Health (K99-CA168996) to N.Z.

\section{References}

Ben-Ami O, Friedman D, Leshkowitz D, Goldenberg D, Orlovsky K, Pencovich N, Lotem J, Tanay A, Groner Y. 2013. Addiction of $\mathrm{t}(8 ; 21)$ and $\operatorname{inv}(16)$ acute myeloid leukemia to native RUNX1. Cell Rep 4: 1131-1143.

Bernt KM, Zhu N, Sinha AU, Vempati S, Faber J, Krivtsov AV, Feng Z, Punt N, Daigle A, Bullinger L, et al. 2011. MLL-rearranged leukemia is dependent on aberrant H3K79 methylation by DOT1L. Cancer Cell 20: 66-78.

Black JC, Van Rechem C, Whetstine JR. 2012. Histone lysine methylation dynamics: establishment, regulation, and biological impact. Mol Cell 48: 491-507.

Brauchle M, Yao Z, Arora R, Thigale S, Clay I, Inverardi B, Fletcher J, Taslimi P, Acker MG, Gerrits B, et al. 2013. Protein complex interactor analysis and differential activity of KDM3 subfamily members towards $\mathrm{H} 3 \mathrm{~K} 9$ methylation. PLoS One 8: e60549.

Cabezas-Wallscheid N, Eichwald V, de Graaf J, Lower M, Lehr HA, Kreft A, Eshkind L, Hildebrandt A, Abassi Y, Heck R, et al. 2013. Instruction of haematopoietic lineage choices, evolution of transcriptional landscapes and cancer stem cell hierarchies derived from an AML1-ETO mouse model. EMBO Mol Med 5: 1804-1820.

Cerchietti LC, Lopes EC, Yang SN, Hatzi K, Bunting KL, Tsikitas LA, Mallik A, Robles AI, Walling J, Varticovski L, et al. 2009. A purine scaffold Hsp90 inhibitor destabilizes BCL- 6 and has specific antitumor activity in BCL-6-dependent B cell lymphomas. Nat Med 15: 1369-1376.

Cerchietti LC, Ghetu AF, Zhu X, Da Silva GF, Zhong S, Matthews M, Bunting KL, Polo JM, Fares C, Arrowsmith CH, et al. 2010. A small-molecule inhibitor of BCL6 kills DLBCL cells in vitro and in vivo. Cancer Cell 17: 400-411.

Chen X, Skutt-Kakaria K, Davison J, Ou YL, Choi E, Malik P, Loeb K, Wood B, Georges G, Torok-Storb B, et al. 2012. G9a/GLP-dependent histone H3K9me2 patterning during human hematopoietic stem cell lineage commitment. Genes Dev 26: 2499-2511.

Daigle SR, Olhava EJ, Therkelsen CA, Majer CR, Sneeringer CJ, Song J, Johnston LD, Scott MP, Smith JJ, Xiao Y, et al. 2011. Selective killing of mixed lineage leukemia cells by a potent small-molecule DOT1L inhibitor. Cancer Cell 20: 53-65.

Dalton WT Jr, Ahearn MJ, McCredie KB, Freireich EJ, Stass SA, Trujillo JM. 1988. HL-60 cell line was derived from a patient with FAB-M2 and not FAB-M3. Blood 71: 242-247.

Dawson MA, Prinjha RK, Dittmann A, Giotopoulos G, Bantscheff M, Chan WI, Robson SC, Chung CW, Hopf C, Savitski $\mathrm{MM}$, et al. 2011. Inhibition of BET recruitment to chromatin as an effective treatment for MLL-fusion leukaemia. Nature 478: 529-533.

DeKelver RC, Yan M, Ahn EY, Shia WJ, Speck NA, Zhang DE. 2013. Attenuation of AML1-ETO cellular dysregulation correlates with increased leukemogenic potential. Blood 121: 3714-3717.

Deshpande AJ, Bradner J, Armstrong SA. 2012. Chromatin modifications as therapeutic targets in MLL-rearranged leukemia. Trends Immunol 33: 563-570.

Dignam JD, Lebovitz RM, Roeder RG. 1983. Accurate transcription initiation by RNA polymerase II in a soluble extract from isolated mammalian nuclei. Nucleic Acids Res 11: 1475-1489.

El Omari K, Hoosdally SJ, Tuladhar K, Karia D, Hall-Ponsele E, Platonova O, Vyas P, Patient R, Porcher C, Mancini EJ. 2013. Structural basis for LMO2-driven recruitment of the SCL:E47bHLH heterodimer to hematopoietic-specific transcriptional targets. Cell Rep 4: 135-147.

Filippakopoulos P, Qi J, Picaud S, Shen Y, Smith WB, Fedorov O, Morse EM, Keates T, Hickman TT, Felletar I, et al. 2010. Selective inhibition of BET bromodomains. Nature 468: 1067-1073.

Gelmetti V, Zhang J, Fanelli M, Minucci S, Pelicci PG, Lazar MA. 1998. Aberrant recruitment of the nuclear receptor corepressor-histone deacetylase complex by the acute myeloid leukemia fusion partner ETO. Mol Cell Biol 18: 7185-7191.

Gorczynski MJ, Grembecka J, Zhou Y, Kong Y, Roudaia L, Douvas MG, Newman M, Bielnicka I, Baber G, Corpora T, et al. 2007. Allosteric inhibition of the protein-protein interaction between the leukemia-associated proteins Runx1 and CBF $\beta$. Chem Biol 14: 1186-1197.

Groppe J, Greenwald J, Wiater E, Rodriguez-Leon J, Economides AN, Kwiatkowski W, Affolter M, Vale WW, Izpisua Belmonte JC, Choe S. 2002. Structural basis of BMP signalling inhibition by the cystine knot protein Noggin. Nature 420: 636-642.

Heidenreich O, Krauter J, Riehle H, Hadwiger P, John M, Heil G, Vornlocher HP, Nordheim A. 2003. AML1/MTG8 oncogene suppression by small interfering RNAs supports myeloid differentiation of $\mathrm{t}(8 ; 21)$-positive leukemic cells. Blood 101: 3157-3163.

Higuchi M, O'Brien D, Kumaravelu P, Lenny N, Yeoh EJ, Downing JR. 2002. Expression of a conditional AML1-ETO oncogene bypasses embryonic lethality and establishes a murine model of human $\mathrm{t}(8 ; 21)$ acute myeloid leukemia. Cancer Cell 1: 63-74.

Kaspers GJ, Zwaan CM. 2007. Pediatric acute myeloid leukemia: towards high-quality cure of all patients. Haematologica 92: 1519-1532.

Kim SM, Kim JY, Choe NW, Cho IH, Kim JR, Kim DW, Seol JE, Lee SE, Kook H, Nam KI, et al. 2010. Regulation of mouse steroidogenesis by WHISTLE and JMJD1C through histone methylation balance. Nucleic Acids Res 38: 6389-6403.

Kruidenier L, Chung CW, Cheng Z, Liddle J, Che K, Joberty G, Bantscheff M, Bountra C, Bridges A, Diallo H, et al. 2012. A selective jumonji H3K27 demethylase inhibitor modulates the proinflammatory macrophage response. Nature 488: 404408.

Laurent B, Ruitu L, Murn J, Hempel K, Ferrao R, Xiang Y, Liu S, Garcia BA, Wu H, Wu F, et al. 2015. A specific LSD1/ KDM1A isoform regulates neuronal differentiation through H3K9 demethylation. Mol Cell 57: 957-970.

Lecuyer E, Hoang T. 2004. SCL: from the origin of hematopoiesis to stem cells and leukemia. Exp Hematol 32: 11-24.

Lee JW, Choi HS, Gyuris J, Brent R, Moore DD. 1995. Two classes of proteins dependent on either the presence or absence of 
thyroid hormone for interaction with the thyroid hormone receptor. Mol Endocrinol 9: 243-254.

Lee MG, Wynder C, Cooch N, Shiekhattar R. 2005. An essential role for CoREST in nucleosomal histone 3 lysine 4 demethylation. Nature 437: 432-435.

Look AT. 1997. Oncogenic transcription factors in the human acute leukemias. Science 278: 1059-1064.

Luo X, Liu Y, Kubicek S, Myllyharju J, Tumber A, Ng S, Che KH, Podoll J, Heightman TD, Oppermann U, et al. 2011. A selective inhibitor and probe of the cellular functions of Jumonji $\mathrm{C}$ domain-containing histone demethylases. I Am Chem Soc 133: 9451-9456.

Lutterbach B, Westendorf JJ, Linggi B, Patten A, Moniwa M, Davie JR, Huynh KD, Bardwell VJ, Lavinsky RM, Rosenfeld MG, et al. 1998. ETO, a target of $\mathrm{t}(8 ; 21)$ in acute leukemia, interacts with the N-CoR and mSin 3 corepressors. Mol Cell Biol 18: 7176-7184.

Martens JH, Mandoli A, Simmer F, Wierenga BJ, Saeed S, Singh AA, Altucci L, Vellenga E, Stunnenberg HG. 2012. ERG and FLI1 binding sites demarcate targets for aberrant epigenetic regulation by AML1-ETO in acute myeloid leukemia. Blood 120: $4038-4048$.

Meng YS, Khoury H, Dick JE, Minden MD. 2005. Oncogenic potential of the transcription factor LYL1 in acute myeloblastic leukemia. Leukemia 19: 1941-1947.

Meyers S, Downing JR, Hiebert SW. 1993. Identification of AML1 and the $(8 ; 21)$ translocation protein (AML-1/ETO) as sequence-specific DNA-binding proteins: the runt homology domain is required for DNA binding and protein-protein interactions. Mol Cell Biol 13: 6336-6345.

Miyamoto T, Nagafuji K, Harada M, Niho Y. 1997. Significance of quantitative analysis of AML1/ETO transcripts in peripheral blood stem cells from $\mathrm{t}(8 ; 21)$ acute myelogenous leukemia. Leuk Lymphoma 25: 69-75.

Mulloy JC, Cammenga J, MacKenzie KL, Berguido FJ, Moore MA, Nimer SD. 2002. The AML1-ETO fusion protein promotes the expansion of human hematopoietic stem cells. Blood 99: $15-23$.

Mulloy JC, Cammenga J, Berguido FJ, Wu K, Zhou P, Comenzo RL, Jhanwar S, Moore MA, Nimer SD. 2003. Maintaining the self-renewal and differentiation potential of human CD34+ hematopoietic cells using a single genetic element. Blood 102: 4369-4376.

Murre C. 2005. Helix-loop-helix proteins and lymphocyte development. Nat Immunol 6: 1079-1086.

Nimer SD, Moore MA. 2004. Effects of the leukemia-associated AML1-ETO protein on hematopoietic stem and progenitor cells. Oncogene 23: 4249-4254.

Ohlsson E, Hasemann MS, Willer A, Lauridsen FK, Rapin N, Jendholm J, Porse BT. 2014. Initiation of MLL-rearranged AML is dependent on C/EBPa. J Exp Med 211: 5-13.

Pabst T, Mueller BU, Harakawa N, Schoch C, Haferlach T, Behre G, Hiddemann W, Zhang DE, Tenen DG. 2001. AML1ETO downregulates the granulocytic differentiation factor C/EBPa in t(8;21) myeloid leukemia. Nat Med 7: 444-451.

Park S, Speck NA, Bushweller JH. 2009. The role of CBF $\beta$ in AML1-ETO's activity. Blood 114: 2849-2850.

Peterson LF, Zhang DE. 2004. The 8;21 translocation in leukemogenesis. Oncogene 23: 4255-4262.

Popovic R, Licht JD. 2012. Emerging epigenetic targets and therapies in cancer medicine. Cancer Discov 2: 405-413.

Ptasinska A, Assi SA, Mannari D, James SR, Williamson D, Dunne J, Hoogenkamp M, Wu M, Care M, McNeill H, et al. 2012. Depletion of RUNX1/ETO in t(8;21) AML cells leads to genome-wide changes in chromatin structure and transcription factor binding. Leukemia 26: 1829-1841.

Roeder RG. 2005. Transcriptional regulation and the role of diverse coactivators in animal cells. FEBS Lett 579: 909-915.

Saeed S, Logie C, Francoijs KJ, Frige G, Romanenghi M, Nielsen FG, Raats L, Shahhoseini M, Huynen M, Altucci L, et al. 2012. Chromatin accessibility, p300, and histone acetylation define PML-RAR $a$ and AML1-ETO binding sites in acute myeloid leukemia. Blood 120: 3058-3068.

Schenk T, Chen WC, Gollner S, Howell L, Jin L, Hebestreit K, Klein HU, Popescu AC, Burnett A, Mills K, et al. 2012. Inhibition of the LSD1 (KDM1A) demethylase reactivates the alltrans-retinoic acid differentiation pathway in acute myeloid leukemia. Nat Med 18: 605-611.

Schessl C, Rawat VP, Cusan M, Deshpande A, Kohl TM, Rosten PM, Spiekermann K, Humphries RK, Schnittger S, Kern W, et al. 2005. The AML1-ETO fusion gene and the FLT3 length mutation collaborate in inducing acute leukemia in mice. $J$ Clin Invest 115: 2159-2168.

Shia WJ, Okumura AJ, Yan M, Sarkeshik A, Lo MC, Matsuura S, Komeno Y, Zhao X, Nimer SD, Yates JR III, et al. 2012. PRMT1 interacts with AML1-ETO to promote its transcriptional activation and progenitor cell proliferative potential. Blood 119: 4953-4962.

Sroczynska P, Cruickshank VA, Bukowski JP, Miyagi S, Bagger FO, Walfridsson J, Schuster MB, Porse B, Helin K. 2014. shRNA screening identifies JMJD1C as being required for leukemia maintenance. Blood 123: 1870-1882.

Sun XJ, Wang Z, Wang L, Jiang Y, Kost N, Soong TD, Chen WY, Tang Z, Nakadai T, Elemento O, et al. 2013. A stable transcription factor complex nucleated by oligomeric AML1ETO controls leukaemogenesis. Nature 500: 93-97.

Tripic T, Deng W, Cheng Y, Zhang Y, Vakoc CR, Gregory GD, Hardison RC, Blobel GA. 2009. SCL and associated proteins distinguish active from repressive GATA transcription factor complexes. Blood 113: 2191-2201.

Vangala RK, Heiss-Neumann MS, Rangatia JS, Singh SM, Schoch C, Tenen DG, Hiddemann W, Behre G. 2003. The myeloid master regulator transcription factor PU.1 is inactivated by AML1-ETO in t $(8 ; 21)$ myeloid leukemia. Blood 101: 270-277.

Visvader J, Begley CG, Adams JM. 1991. Differential expression of the LYL, SCL and E2A helix-loop-helix genes within the hemopoietic system. Oncogene 6: 187-194.

Wadman IA, Osada H, Grutz GG, Agulnick AD, Westphal H, Forster A, Rabbitts TH. 1997. The LIM-only protein Lmo2 is a bridging molecule assembling an erythroid, DNA-binding complex which includes the TAL1, E47, GATA-1 and Ldb1/ NLI proteins. EMBO J 16: 3145-3157.

Wang J, Hoshino T, Redner RL, Kajigaya S, Liu JM. 1998. ETO, fusion partner in $\mathrm{t}(8 ; 21)$ acute myeloid leukemia, represses transcription by interaction with the human $\mathrm{N}-\mathrm{CoR} / \mathrm{mSin} 3 /$ HDAC1 complex. Proc Natl Acad Sci 95: 10860-10865.

Wang L, Gural A, Sun XJ, Zhao X, Perna F, Huang G, Hatlen MA, Vu L, Liu F, Xu H, et al. 2011a. The leukemogenicity of AML1ETO is dependent on site-specific lysine acetylation. Science 333: 765-769.

Wang YY, Zhao LJ, Wu CF, Liu P, Shi L, Liang Y, Xiong SM, Mi JQ, Chen Z, Ren R, et al. 2011b. C-KIT mutation cooperates with full-length AML1-ETO to induce acute myeloid leukemia in mice. Proc Natl Acad Sci 108: 2450-2455.

Watanabe S, Watanabe K, Akimov V, Bartkova J, Blagoev B, Lukas J, Bartek J. 2013. JMJD1C demethylates MDC1 to regulate the RNF8 and BRCA1-mediated chromatin response to DNA breaks. Nat Struct Mol Biol 20: 1425-1433. 
Wilson NK, Foster SD, Wang X, Knezevic K, Schutte J, Kaimakis P, Chilarska PM, Kinston S, Ouwehand WH, Dzierzak E, et al. 2010. Combinatorial transcriptional control in blood stem/ progenitor cells: genome-wide analysis of ten major transcriptional regulators. Cell Stem Cell 7: 532-544.

Wolf SS, Patchev VK, Obendorf M. 2007. A novel variant of the putative demethylase gene, s-JMJD1C, is a coactivator of the AR. Arch Biochem Biophys 460: 56-66.

Yamane K, Toumazou C, Tsukada Y, Erdjument-Bromage H, Tempst P, Wong J, Zhang Y. 2006. JHDM2A, a JmjC-containing $\mathrm{H} 3 \mathrm{~K} 9$ demethylase, facilitates transcription activation by androgen receptor. Cell 125: 483-495.

Yan M, Burel SA, Peterson LF, Kanbe E, Iwasaki H, Boyapati A, Hines R, Akashi K, Zhang DE. 2004. Deletion of an AML1ETO C-terminal NcoR/SMRT-interacting region strongly induces leukemia development. Proc Natl Acad Sci 101: 17186-17191.
Yan M, Kanbe E, Peterson LF, Boyapati A, Miao Y, Wang Y, Chen IM, Chen Z, Rowley JD, Willman CL, et al. 2006. A previously unidentified alternatively spliced isoform of $t$ $(8 ; 21)$ transcript promotes leukemogenesis. Nat Med 12: 945-949.

Zhang J, Kalkum M, Yamamura S, Chait BT, Roeder RG. 2004. E protein silencing by the leukemogenic AML1-ETO fusion protein. Science 305: 1286-1289.

Zhou J, Wu J, Li B, Liu D, Yu J, Yan X, Zheng S, Wang J, Zhang L, He F, et al. 2014. PU.1 is essential for MLL leukemia partially via crosstalk with the MEIS/HOX pathway. Leukemia 28: 1436-1448.

Zuber J, Shi J, Wang E, Rappaport AR, Herrmann H, Sison EA, Magoon D, Qi J, Blatt K, Wunderlich M, et al. 2011. RNAi screen identifies Brd4 as a therapeutic target in acute myeloid leukaemia. Nature 478: 524-528. 


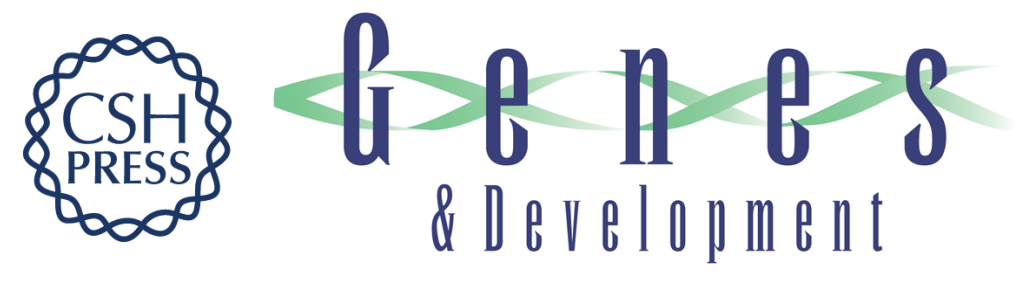

\section{JMJD1C is required for the survival of acute myeloid leukemia by functioning as a coactivator for key transcription factors}

Mo Chen, Nan Zhu, Xiaochuan Liu, et al.

Genes Dev. 2015, 29:

Access the most recent version at doi:10.1101/gad.267278.115

\section{Supplemental http://genesdev.cshlp.org/content/suppl/2015/10/22/29.20.2123.DC1 \\ Material}

References This article cites 68 articles, 27 of which can be accessed free at:

http://genesdev.cshlp.org/content/29/20/2123.full.html\#ref-list-1

Creative This article is distributed exclusively by Cold Spring Harbor Laboratory Press for the first

Commons six months after the full-issue publication date (see

License http://genesdev.cshlp.org/site/misc/terms.xhtml). After six months, it is available under a Creative Commons License (Attribution-NonCommercial 4.0 International), as described at http://creativecommons.org/licenses/by-nc/4.0/.

Email Alerting Receive free email alerts when new articles cite this article - sign up in the box at the top Service right corner of the article or click here.

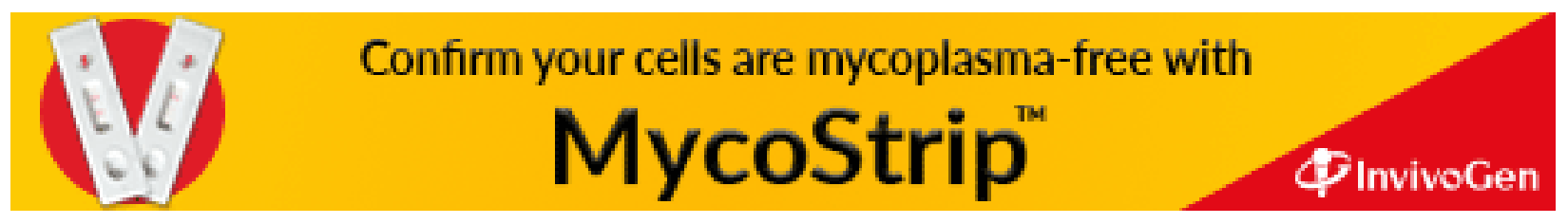

